\title{
Análisis de la producción y redes de colaboración en los programas de doctorado en psicología en Colombia
}

\author{
Wilson López-López; Julio César Ossa; Jean Nikola Cudina; María Constanza Aguilar Bustamante; \\ Michelle Torres; Cesar Acevedo-Triana; Gonzalo Salas
}

Cómo citar este artículo:

López-López, W., Ossa, J. C., Cudina, J. N., Aguilar, M. C., Torres, M., Acevedo-Triana, C., \& Salas, G. (2022). Análisis de la producción y redes de colaboración en los programas de doctorado en psicología en Colombia. Acta Colombiana de Psicología, 25(1), 151-182. https://www.doi.org/10.14718/ACP.2022.25.1.11

Recibido, septiembre 30/2020; Concepto de evaluación, octubre 29/2021; Aceptado, noviembre 2/2021

\author{
Wilson López-López ${ }^{1}$ \\ ORCID: https://orcid.org/0000-0002-2964-0402 \\ Pontificia Universidad Javeriana, Bogotá, Colombia. \\ Julio César Ossa \\ ORCID: https://orcid.org/0000-0002-3079-3318 \\ Fundación Universitaria de Popayán, Popayán, Colombia. \\ Jean Nikola Cudina \\ ORCID: https://orcid.org/0000-0003-4004-7615 \\ Fundación Universitaria Lumen Gentium, Cali, Colombia. \\ María Constanza Aguilar Bustamante \\ ORCID: https://orcid.org/0000-0003-2990-9667 \\ Universidad Católica de Colombia, Bogotá, Colombia. \\ Michelle Torres \\ ORCID: https://orcid.org/0000-0003-4209-0342 \\ Universidad Pedagógica y Tecnológica de Colombia, Tunja, Boyacá. \\ Cesar Acevedo-Triana \\ ORCID: https://orcid.org/0000-0002-1296-9957 \\ Universidad Pedagógica y Tecnológica de Colombia. \\ Gonzalo Salas \\ ORCID: https://orcid.org/0000-0003-0707-8188 \\ Universidad Católica del Maule, Talca, Chile.
}

\begin{abstract}
Resumen
El objetivo de la formación doctoral es la generación y difusión de nuevo conocimiento, sin embargo, no es claro cómo los programas de doctorado en Colombia se relacionan con este tipo de producción académica. A partir de ello, en el presente estudio se presenta el panorama general de la producción académica a través de las instituciones de educación superior colombianas que ofertan programas de formación doctoral en psicología. La producción académica se obtuvo de la base de datos Scopus en una muestra de 13 universidades. Tras una búsqueda inicial, se encontraron 1345 documentos, con los cuales se realizó un análisis de los indicadores de productividad y de las redes de colaboración al interior de la disciplina en el país. Posteriormente, esta información se contrastó con la información nacional registrada en el sistema de Currículum Vitae Latinoamericano y del Caribe (CvLAC) de 193 profesores asociados a las universidades que ofertan los programas de doctorado. Al comparar la producción académica nacional e internacional, se encontró una mayor correlación entre la dirección de tesis de posgrado y la publicación en revistas nacionales. Por lo tanto, se puede afirmar que el impacto de la formación doctoral en la generación de nuevo conocimiento es relativo y tiene un alcance limitado, siendo principalmente las revistas nacionales el medio de difusión de este conocimiento. Se recomienda evaluar el impacto de los productos asociados a la formación posgradual y la visibilidad internacional, con énfasis en las revistas indexadas en bases de datos internacionales. Palabras clave: producción académica, programas de formación doctoral, Colombia.
\end{abstract}

1 Cra 5 \# 39-00, piso 2, Edificio Manuel Briceño, Pontificia Universidad Javeriana, Bogotá, Colombia. Tel.: 3208320 ext. 5757. lopezw@javeriana.edu.co

Este artículo forma parte del proyecto SIAP de la Pontifica Universidad Javeriana (9608). 


\title{
Analysis of production and collaboration networks in doctoral programs in psychology in Colombia
}

\begin{abstract}
The objective of doctoral training is the generation and dissemination of new knowledge; however, it is not clear how doctoral programs in Colombia relate to this type of production. Based on this, this study presents the general panorama of academic production across Colombian higher education institutions that offer doctoral training programs in psychology. The academic production was obtained from the Scopus database in a sample of 13 universities. After this search, 1345 documents were found with which an analysis was made regarding productivity indicators and the collaboration networks within the discipline in the country were identified. Subsequently, this information was contrasted with the national information registered in the Latin American and Caribbean Curriculum Vitae system (CvLAC) of 193 professors associated with the universities offering doctoral programs. As a result, when comparing national and international academic production, a greater correlation is found between the supervision of graduate theses and publication in national journals. In this sense, it is found that the impact of doctoral training on the generation of new knowledge is relative and has a limited scope, being mainly national journals the means of dissemination of this knowledge. It is recommended to evaluate the impact of products associated with postgraduate training and international visibility, with emphasis on indexed journals in international databases.

Keywords: academic production, doctoral training programs, Colombia.
\end{abstract}

\section{Introducción}

La producción de conocimiento en la ciencia, y particularmente en psicología, es principalmente derivada de la investigación realizada en los programas de posgrado (García-Martínez et al., 2012; Kamler, 2008; Lafont, 2014). Esta producción científica, al igual que la oferta de formación doctoral en Colombia, ha aumentado en los últimos 15 años, lo cual ha tenido como consecuencia una mayor visibilidad en la investigación en diferentes campos académicos (Acevedo-Triana et al., 2018; Moya Anegon, 2011; Salazar-Acosta et al., 2013). Para entender las particularidades del fenómeno de las publicaciones y los campos de conocimiento, es necesario reconocer los diversos factores que intervienen y deben ser abordados, como lo son, por ejemplo, el crecimiento de las instituciones, el aumento y la cohesión de la comunidad académica, el contexto social y cultural, el contexto histórico, el aumento o disminución de la financiación, y el incremento en la producción y difusión de este nuevo conocimiento. Estos elementos pueden abordarse de forma global en el concepto de ecosistema de conocimiento, ya que en este se reconoce el aporte y peso de cada elemento durante el proceso de investigaciónpublicación (López-López, 2019a, 2019b, 2020).

En particular, el ecosistema de conocimiento en psicología en Colombia ha experimentado profundos cambios en los últimos años (López-López et al., 2015), principalmente reflejados en la infraestructura de las instituciones y los programas, en los sistemas de incentivos en cuanto a la publicación, en las exigencias respecto a los procesos de apropiación de conocimiento, en los procesos en el marco de las publicaciones y los investigadores, y en la comunidad que recibe el conocimiento producido (López-López, 2019a, 2020).

Además de los elementos tradicionales en el proceso de investigación-publicación, el concepto de ecosistema de conocimiento también contempla los cambios en las políticas públicas de ciencia y tecnología que modifican las estructuras institucionales, su gobernanza, los actores involucrados, los procesos de investigación, formación, comunicación, difusión e innovación, y las dinámicas de cooperación y transferencia de conocimiento (LópezLópez, 2018).

En el caso colombiano, desafortunadamente la inversión en investigación y desarrollo ha disminuido en el periodo de 2014 a 2018, pues, según los datos de libre acceso del Banco Mundial (2021), específicamente en los indicadores de gasto en investigación y desarrollo, se encuentra que esta pasó del $0.3 \%$ del producto interno bruto (PIB) en $2014 \mathrm{al}$ $0.23 \%$ en 2018, por debajo de países como Brasil (1.26\%), Argentina ( $0.54 \%)$, Chile (0.35\%) y México (0.31\%), lo que ha impactado negativamente este ecosistema. Sumado 
a esto, la medición de la producción en los diferentes contextos y los indicadores de indexación y acreditación han ejercido presión sobre el aumento en los niveles de publicación y un cambio en las políticas de producción y visibilidad, lo cual ha terminado comprometiendo el valor ético y social de los ecosistemas de conocimiento (Ana et al., 2013; Ayodele et al., 2019; Di Bitetti \& Ferreras, 2017; Vasconcelos et al., 2009).

En cuanto a la evaluación y medición de varios de estos elementos del ecosistema, podemos encontrar distintos indicadores de medición de los procesos y los productos científicos (Bornmann et al., 2021; Bustos-González, 2019; López-López, 2018). En particular, en el campo de la psicología, son múltiples los estudios bibliométricos y cienciométricos que dan cuenta de dinámicas de producción, cooperación y comunicación (Acevedo-Triana et al., 2018; Gallegos et al., 2020; Guerrero \& Jaraba, 2009; JarabaBarrios et al., 2011; López-López et al., 2010; Morales et al., 2012; Ossa \& Cudina, 2016; Vera-Villarroel et al., 2011). Recientemente, algunos de estos estudios han permitido identificar cambios del ecosistema por medio del análisis de las redes sociales (Ávila-Toscano et al., 2014; Cudina et al., 2017), una perspectiva que favorece el estudio de las interacciones entre los actores del ecosistema, en el marco de un componente cognitivo y social de la ciencia (Gómez, 2017).

En este mismo sentido, se han reportado procesos particulares de comunicación científica y de evaluación del crecimiento y rendimiento de la producción académica a través de políticas públicas de ciencia y tecnología (Vélez-Cuartas et al., 2014); así como el reconocimiento de investigadores y comunidades académicas por conocer el posicionamiento de su área de especialización (Vasconcelos et al., 2008). La naturaleza de este tipo de estudios ha generado en la psicología colombiana - en función de su desarrollo histórico - que tenga un conocimiento actualizado de los indicadores de producción sobre la investigación psicológica de Colombia y la región (López-López et al., 2018; Peña-Correal, 1993).

En general, el análisis de la producción científica de la psicología en Colombia se ha realizado principalmente con técnicas de bibliometría descriptiva (Gallegos et al., 2020), y dichos estudios han incluido desde el análisis de la publicación en revistas especializadas (Ravelo-Contreras et al., 2020; Rivera-Garzón, 2008; Salas et al., 2019) hasta el análisis de redes de comunicación científica para develar cómo se conforman las estructuras y comunidades académicas, y su un impacto en la colaboración (Aguilar Bustamante \& Aguado López, 2018; García et al., 2017; Miguel et al., 2012). Asimismo, se han realizado estudios sobre el análisis de los patrones de citación para identificar el impacto de las publicaciones académicas e investigadores, así como para conocer los patrones de comportamiento que se establecen al interior de la disciplina (Aguado-López et al., 2009, 2016; Russell et al., 2007).

Aunque existe una amplia diversidad en las métricas tradicionalmente propuestas, no se tienen indicadores que identifiquen la relación entre los programas de doctorado en psicología y la producción académica en psicología en Colombia; una relación que es relevante si se tiene en cuenta que el crecimiento de los programas de posgrado debería haber ido de la mano con el aumento en la investigación $\mathrm{y}$, por ende, en la producción académica (Alzate Medina, 2008; López-López et al., 2018).

Al respecto, algunos estudios han mostrado que los sistemas de acreditación han mejorado los indicadores de producción de las instituciones académicas (Aguilar Bustamante, 2018; Alzate Medina, 2008); sin embargo, también es clara la falencia de articulación entre los programas y la generación de nuevo conocimiento derivado de estos procesos de investigación (Calderón-Prada \& Cuartas-Arias, 2012). Ahora bien, esta falencia en el ecosistema de conocimiento no solo se debe a los actores, sino que también se refleja en el tipo de instituciones que ofertan estudios de posgrado, en contraste con aquellas más dedicadas a una labor docente y con menos intención de investigación y, por ende, menor inversión de recursos en investigación (Bustos-González, 2019).

Teniendo lo anterior en cuenta, una aproximación para la articulación de este escenario es centrar la investigación en un ecosistema de conocimiento en donde se evalúe integralmente todo el proceso de investigación y producción, y un primer insumo para entender la relación entre estos diferentes actores es el análisis del aumento de los programas de formación doctoral y la producción derivada de dichos programas.

Por esta razón, el objetivo del presente estudio fue responder a la pregunta: ¿cómo contribuyen los programas de formación doctoral con la generación de conocimiento en Colombia? Para dar una respuesta a este interrogante, en el 
presente trabajo se realizó, inicialmente, un balance de las redes nacionales e internacionales de colaboración científica en Colombia en las últimas dos décadas; posteriormente, se estudió el avance, los logros y los desafíos de las revistas de psicología a nivel nacional con el fin de visibilizar el desarrollo de la investigación psicológica en Colombia en el contexto internacional; después, se analizaron algunos indicadores de producción académica respecto a la investigación que se está desarrollando en las instituciones de educación superior que cuentan con la oferta de programas académicos de doctorado —en este punto se realizó un contraste entre la información reportada nacionalmente y la información internacional-; y, finalmente, se buscó aportar elementos para el debate respecto al impacto que tiene el sistema de acreditación de alta calidad (CNA) en los programas de formación doctoral en Colombia.

\section{Un balance en la investigación psicológica en Colombia}

La investigación en psicología en Colombia ha tenido un importante desarrollo y crecimiento, sustentado en el aumento de la producción académica en los últimos 20 años (Acevedo-Triana et al., 2018; Giraldo \& Rodríguez, 2000; Guerrero \& Jaraba, 2009; Jaraba-Barrios et al., 2011; PucheNavarro \& Ossa, 2012). La naturaleza de este crecimiento ha permitido identificar indicadores propios de las dinámicas de investigación de la disciplina, que se corresponden con eventos importantes en el crecimiento de los programas, el aumento de las publicaciones, la generación de sistemas de medición alternativos que dan cuenta de la producción a nivel regional, y el surgimiento en modelos de revistas de acceso abierto propios de América Latina (Alperin, 2014, 2015; Piwowar et al., 2018; Vessuri, 2014).

Por una parte, la evidencia empírica muestra que las dinámicas de producción académica al interior de la disciplina tienden hacia el trabajo colaborativo entre diversos académicos en el ámbito nacional e internacional (Aguado-López et al., 2009, 2016, 2017; Russell et al., 2007). En campos cercanos a la psicología se ha mostrado que la presencia de psicólogos a nivel nacional que contribuyen con investigaciones publicadas en revistas internacionales de alto impacto es un acontecimiento decisivo que favorece las distintas áreas disciplinares (Forero et al., 2020; Robayo-Castro et al., 2016). Además, el impacto de la investigación psicológica en términos de citación ha comenzado a mostrar un crecimiento importante
(Cudina \& Ossa, 2016). Avances que pueden entenderse como un resultado decisivo en el proceso de posicionar la investigación psicológica en el país.

Parte de este crecimiento se debe a un fortalecimiento de la infraestructura científica y de la comunidad académica, la cual, aunque con una inversión modesta desde el ámbito público, cuenta con un compromiso profesional por parte de los investigadores que dinamizan y promueven el desarrollo de la investigación psicológica en Colombia (Garcia et al., 2015; López-López et al., 2015). Asimismo, este aumento en el fortalecimiento de ecosistema científico se debe a la consolidación de la infraestructura tanto en función de las revistas especializadas como de las comunidades académicas, las instituciones de educación superior, los programas de formación, y las políticas para el fomento de la ciencia y tecnología (Alzate Medina, 2008; García et al., 2017; Gutiérrez \& Landeira-Fernández, 2018; López-López et al., 2010; Rivera-Garzón, 2008). Todas estas son una muestra del crecimiento en la investigación psicológica en Colombia, la cual representa a su vez un aporte importante a la disciplina en la región.

Específicamente, estos hechos se evidencian en el aumento de las revistas nacionales indexadas en diversas bases de datos a nivel internacional, lo cual ha permitido mejorar de manera significativa la visibilidad y la calidad editorial y científica de las publicaciones en lo corrido de la última década (Ávila-Toscano \& Rambal-Rivaldo, 2020; Franco-Suárez \& Quevedo-Blasco, 2015; López-López et al., 2015). De igual forma, destaca la conformación de comunidades académicas activas en los diferentes campos de intervención y aplicación de la disciplina, así como el crecimiento en los índices de colaboración y cooperación de la investigación psicológica del país con los demás países de la región, Norteamérica y Europa (Ávila-Toscano et al., 2019; Calderón-Prada \& Cuartas-Arias, 2012; Fernández et al., 1998; Gallegos et al., 2014; García et al., 2014; Garcia et al., 2017).

Es importante reconocer que no es fortuito que estos indicadores muestren un avance importante al interior de la psicología colombiana. En parte, esto ha sido posible gracias del fomento de políticas de ciencia y tecnología y de educación superior para estimular el desarrollo de la investigación de calidad en el país (Chavarro et al., 2010; López-López et al., 2018; Orozco et al., 2013). De hecho, esta política nacional para el fomento de la ciencia 
y tecnología del país ha promovido una agenda de trabajo ambiciosa que contribuye al proyecto de visibilizar la producción académica local. Igualmente, se puede evidenciar que en los últimos años el crecimiento investigativo a nivel regional también ha potenciado el crecimiento de la investigación en Colombia.

\section{Método}

\section{Diseño}

El presente estudio se realizó a partir de un diseño descriptivo y mediante un análisis documental (Barbosa et al., 2013). Con este, se reporta la producción de investigación en psicología en Colombia por medio del análisis de distintos indicadores de producción académica.

\section{Unidades de análisis}

Por una parte, el análisis realizado en este estudio se concentró en los documentos de la producción académica indexada en Scopus derivada de las instituciones de educación superior del país que contaban con programas académicos de formación doctoral en psicología hasta el año 2020; y en la información de los documentos — artículos o libros-, revistas —nacionales o internacionales-y trabajos de grado dirigidos por los investigadores asociados a dichos programas, reportados en el CvLAC (Curriculum Vitae de Latinoamérica y el Caribe $)^{1}$ de cada investigador.

El análisis se dirigió hacia cuatro objetivos: en primer lugar, caracterizar la producción académica y, con esto, establecer indicadores de impacto y consumo de la investigación psicológica en Colombia; en segundo lugar, identificar las redes de colaboración nacional e internacional que se tejen en la comunidad académica de psicólogos e instituciones de educación superior del país; en tercer lugar, exponer algunos datos que reflejen el panorama de la formación doctoral en psicología en el país; $y$, finalmente, recolectar la información reportada en los CvLAC de cada investigador con el fin de contrastar esta producción con la

\footnotetext{
1 La consulta de los CvLAC se realizó en las fechas en las que típicamente los investigadores actualizan y verifican la información reportada, debido a la convocatoria de medición y clasificación de grupos de investigación por parte del Ministerio de Ciencia y Tecnología, antiguo Colciencias.
}

reportada en Scopus. En general, esta investigación tiene como propósito incitar al debate en torno a la dinámica de investigación, producción y difusión de la investigación en psicología en Colombia.

\section{Procedimiento}

El procedimiento para el análisis de los datos y resultados que se exponen en este estudio se llevó a cabo a partir de cuatro fases:

Fase 1: identificación de los programas de formación doctoral en psicología del país a través de la plataforma SNIES (Sistema Nacional de Información de la Educación Superior, del Ministerio de Educación de Colombia ${ }^{2}$ ), con lo cual se obtuvieron en total 13 instituciones de educación superior (IES) que cumplían con el requisito de contar con un doctorado en psicología. La producción académica indexada en el área de psicología - de cada una de estas instituciones educativas se extrajo de la base de datos Scopus.

Fase 2: consulta del registro de hoja vida - CvLAC - de cada uno de los investigadores asociados a los programas de doctorado de las 13 IEs identificadas anteriormente. En la mayoría de los casos, las IES indicaban claramente los profesores asociados a estos programas, pero en los casos en los que no se identificó claramente a los profesores asociados al programa de doctorado, se consultó la información de todos los profesores asociados al programa de psicología, tanto de pregrado como de posgrado.

Fase 3: depuración los datos en las tablas de registro. La información básica incluyó metadatos de autores - afiliación institucional, frecuencia de publicaciones, frecuencia de citas e índice $\mathrm{H}-\mathrm{y}$ de artículos — año de publicación, revista de publicación, información de coautoría-. Para la información de la base de datos de Scopus, el criterio de selección se basó en los artículos de investigadores que firman con las afiliaciones de las IES seleccionadas. Con respecto a los datos del CvLAC, la información se extrajo del aparatado de publicaciones —artículos y libros-, de la consulta de las revistas de dichos escritos - nacionales o internacionales - y de trabajos de posgrado dirigidos.

Fase 4: análisis de datos. En lo que respecta a los indicadores producción de la psicología en Colombia, se analizaron los datos relacionados con el impacto y consumo de la producción académica a través del análisis de citación

\footnotetext{
Disponible en https://snies.mineducacion.gov.co/portal/
} 
(White \& White, 1977). También, se analizaron los datos de los académicos con mayor productividad en los diversos campos de investigación al interior de la disciplina; $\mathrm{y}$, por otro lado, se identificaron las redes de colaboración a partir del análisis de redes (Otte \& Rousseau, 2002). Las representaciones graficas de los mapas expuestos en este estudio se llevaron a cabo con la asistencia del software Vosviewer (van Eck \& Waltman, 2010).

\section{Resultados}

De acuerdo con el Sistema Nacional de Información de la Educación Superior (SNIES), en Colombia existen 13 instituciones de educación superior que ofertan programas de formación doctoral en psicología. En total, el corpus empírico que se desprende de la producción académica de estas universidades es de 1343 documentos indexados en Scopus. De estos, el $88.6 \%(n=1190)$ corresponde a la tipología de artículos de investigación, y el $11.4 \%$ restante de la producción académica se clasificó como artículos de revisión $(n=71)$, editoriales $(n=39)$, capítulos de libro $(n=25)$, artículos de conferencia $(n=6)$, notas $(n=4)$; libros $(n=3)$, erratas $(n=2)$, reportes breves $(n=2) \mathrm{y}$ cartas $(n=1)$.

A continuación se presenta el análisis de esta producción en función de cuatro apartados generales, a saber: (a) programas de doctorado en Colombia; (b) indicadores en la producción académica de la psicología en Colombia; (c) redes de colaboración de la investigación psicológica en Colombia; y (d) producción reportada en CvLAC.

\section{Programas de doctorado en Colombia}

La formación doctoral en psicología en el país cuenta con una historia relativamente reciente. Pese a esto, la oferta de programas académicos de doctorado en lo corrido del último quinquenio ha tenido avances importantes desde la creación del primer doctorado en la Universidad del Valle en el año 2004; avances que se sustentan en la apertura de nuevos programas académicos, consecuentes con la visibilidad y el crecimiento de la investigación en psicología en el país.

Según el SNIES (2020), existen en total 365 programas de doctorado en Colombia - consulta realizada en el SNIES de Colombia, en diciembre de 2020 - La clasificación de los programas académicos de doctorado según el área de conocimiento se presenta en la Figura 1. Específicamente, como se observa en la Figura 2, existe una gran diversidad en la formación según su núcleo básico del conocimiento dentro de los 90 programas del área de conocimiento de las ciencias sociales y humanas.

En particular, de acuerdo con cifras del SNIES (2020), en Colombia se ofertan 16 programas de doctorado en psicología, 3 ofertados por universidades del sector oficial y 13 por universidades del sector privado. Según el nombre del programa registrado en SNIES, 12 doctorados están registrados bajo el nombre de Doctorado en Psicología, y los 4 restantes responden a un nombre con mayor especificidad:

Figura 1.

Oferta de programas académicos de doctorado por área de conocimiento en Colombia

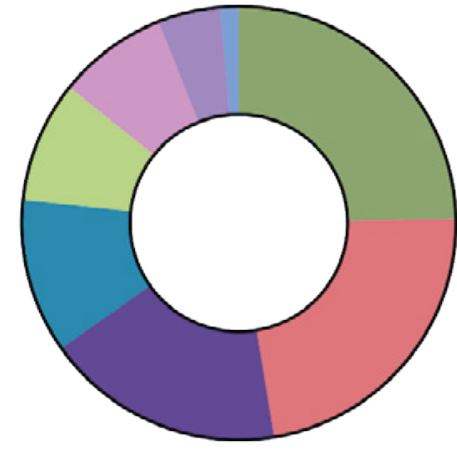

Total $=365$
90 Ciencias sociales y humanas

83 Ingeniería, arquitectura, urbanismo

$\square 65$ Matemáticas y ciencias naturales

$\square 42$ Ciencias de la salud

$\square 33$ Economía, administración, contaduría

$\square 30$ Ciencias de la educación

$\square 17$ Agronomía, veterinaria y afines

5 Bellas artes

Fuente: elaboración propia. 
Figura 2.

Núcleo básico de conocimiento en programas de doctorado de ciencias sociales y humanas

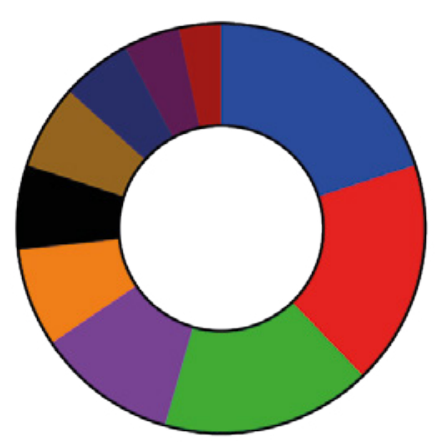

Total $=90$

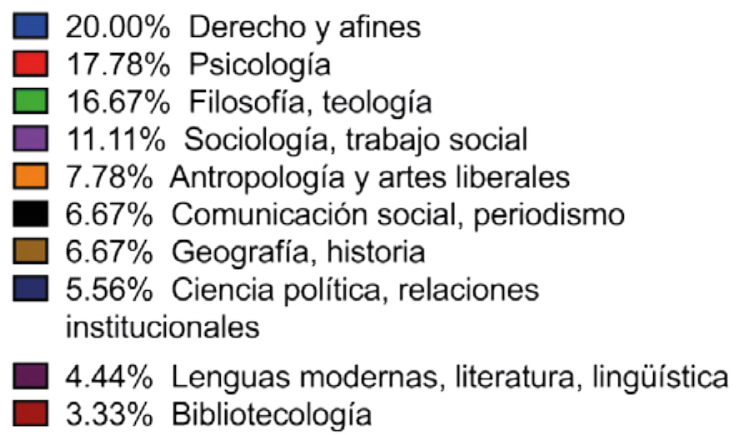

$\square 20.00 \%$ Derecho y afines

$\square 11.11 \%$ Sociologia, trabajo social

$\square 7.78 \%$ Antropología y artes liberales

$6.67 \%$ Comunicación social, periodismo

$6.67 \%$ Geografía, historia

$4.44 \%$ Lenguas modern
$3.33 \%$ Bibliotecología

Fuente: elaboración propia.

Doctorado en Psicoanálisis, Doctorado en Neurociencia Cognitiva Aplicada, Doctorado en Neurociencia Aplicada y Comportamiento, y Doctorado en Estudios Interdisciplinarios (véase Tabla 1).

De la Tabla 1 es importante destacar la cronología en la fecha de registro de cada programa en el SNIES. Como vemos, el primer registro fue el realizado por el programa de doctorado de la Universidad del Valle en el año 2004, y el más reciente fue el realizado por el programa de la Universidad Católica Luis Amigó-Funlam en el año 2020.
Asimismo, es de destacar la oferta de programas académicos en el último quinquenio, teniendo en cuenta que el $58 \%$ de los programas académicos se han ofertado en este periodo. De igual forma, es importante resaltar que los títulos otorgados en estos programas son en psicología en general, y que estos incluyen líneas de investigación en diferentes áreas — como neuropsicología, desarrollo, aprendizaje o cognición-, dependiendo de las líneas de investigación que se derivan de la especialidad de los grupos de investigación, a excepción del programa de la

Tabla 1.

Instituciones de educación superior que ofertan programas de doctorado

\begin{tabular}{lcc}
\hline \multicolumn{1}{c}{ Nombre del programa } & Institución que los oferta & Fecha de registro \\
\hline Doctorado en Psicología & $*$ Universidad del Valle & 2004 \\
Doctorado en Psicología & Universidad del Norte & 2005 \\
Doctorado en Psicología & Universidad de Los Andes & 2008 \\
Doctorado en Psicología & Universidad de San Buenaventura, Medellín & 2010 \\
Doctorado en Psicología & $*$ Universidad Nacional de Colombia & 2014 \\
Doctorado en Psicoanálisis & $*$ Universidad de Antioquia & 2015 \\
Doctorado en Psicología & Universidad Simón Bolívar & 2016 \\
Doctorado en Psicología & Universidad de San Buenaventura, Cali \\
Doctorado en Neurociencia Cognitiva Aplicada & Corporación Universidad de la Costa \\
Doctorado en Psicología & Fundación Universitaria Konrad Lorenz \\
Doctorado en Psicología & Pontificia Universidad Javeriana, Bogotá \\
Doctorado en Psicología & Pontificia Universidad Javeriana, Cali & 2016 \\
Doctorado en Psicología & Universidad Católica de Colombia & 2016 \\
Doctorado en Neurociencia Aplicada y Comportamiento & Universidad de San Buenaventura, Bogotá \\
Doctorado en Psicología & Universidad Santo Tomás \\
Doctorado en Estudios Interdisciplinarios en Psicología & Universidad Católica Luis Amigó-Funlam & 2016 \\
\hline
\end{tabular}

Nota. ${ }^{*}$ Universidades del sector oficial. 
Universidad Católica Luis Amigó, que otorga un título en Estudios Interdisciplinarios en Psicología.

Por otra parte, de acuerdo con el SNIES (2020), en Colombia hay 360 instituciones educativas, 87 de las cuales cuentan con acreditación de alta calidad (24\%). Según el carácter académico de dichas instituciones, se encuentran 29 instituciones técnicas profesionales, ninguna de ellas acreditadas con alta calidad; 53 instituciones tecnológicas, 2 de ellas acreditadas con alta calidad (lo cual representa solo el $3.8 \%$ ); 139 instituciones universitarias, 9 de ellas acreditadas con alta calidad (esto representa el $6.5 \%$ ); y 139 universidades, 76 de ellas acreditadas con alta calidad (esto es el 54.7\%).

De lo anterior, cabe destacar que el $84.6 \%$ de las instituciones educativas que ofertan programas de formación doctoral cuentan con el reconocimiento de alta calidad del Ministerio de Educación. En lo que respecta a los programas de psicología (pregrado), se encuentra que 48 de estos cuentan con acreditación de alta calidad (esto representa el $35.6 \%$ ) y 87 con registro calificado (64.4\%). Finalmente, es importante resaltar que 16 de los programas de psicología que ofertan programas de formación doctoral cuentan con acreditación de alta calidad (véase Tabla 2).

En este sentido, resalta el aporte y respaldo que los programas de psicología brindan para la formulación de un programa de doctorado, y es importante destacar que en su totalidad son programas que cuentan con la acreditación de alta calidad. Al respecto, el Ministerio de Educación (2019) afirma que el Sistema de Aseguramiento de la Calidad de

Tabla 2.

Reconocimiento del ministerio al programa de doctorado al plan de estudios y a las instituciones que ofertan programas de doctorado en Colombia

\begin{tabular}{|c|c|c|c|}
\hline Institución que oferta programa de doctorado & $\begin{array}{c}\text { Institución } \\
\text { educativa }\end{array}$ & Programa de psicología & Programa de doctorado \\
\hline *Universidad del Valle & $\begin{array}{c}\text { Alta calidad } \\
\text { (10 años) }\end{array}$ & $\begin{array}{l}\text { Alta calidad } \\
\text { (8 años) }\end{array}$ & Acreditación de alta calidad \\
\hline Universidad del Norte & $\begin{array}{l}\text { Alta calidad } \\
\text { (8 años) }\end{array}$ & $\begin{array}{l}\text { Alta calidad } \\
\text { (8 años) }\end{array}$ & Acreditación de alta calidad \\
\hline Universidad de Los Andes & $\begin{array}{l}\text { Alta calidad } \\
\text { (10 años) }\end{array}$ & $\begin{array}{l}\text { Alta calidad } \\
\text { (8 años) }\end{array}$ & Registro calificado \\
\hline Universidad de San Buenaventura & $\begin{array}{l}\text { Alta calidad } \\
\text { (4 años) }\end{array}$ & $\begin{array}{c}\text { Alta calidad } \\
\text { (Bogotá } 8,5 \text { años) } \\
\text { (Cali } 6 \text { años) } \\
\text { (Medellín } 6 \text { años) }\end{array}$ & Registro calificado \\
\hline *Universidad Nacional de Colombia & $\begin{array}{c}\text { Alta calidad } \\
\text { (10 años) }\end{array}$ & $\begin{array}{l}\text { Alta calidad } \\
\text { (8 años })\end{array}$ & Registro calificado \\
\hline *Universidad de Antioquia & $\begin{array}{l}\text { Alta calidad } \\
\text { (10 años) }\end{array}$ & $\begin{array}{l}\text { Alta calidad } \\
\text { (Medellín } 6 \text { años) }\end{array}$ & Registro calificado \\
\hline Universidad Simón Bolívar & $\begin{array}{l}\text { Alta calidad } \\
\text { (4 años })\end{array}$ & $\begin{array}{c}\text { Alta calidad } \\
\text { (Barranquilla } 8,5 \text { años) }\end{array}$ & Registro calificado \\
\hline Corporación Universidad de la Costa & $\begin{array}{l}\text { Alta calidad } \\
\text { (4 años })\end{array}$ & $\begin{array}{l}\text { Alta calidad } \\
\text { (4 años })\end{array}$ & Registro calificado \\
\hline Fundación Universitaria Konrad Lorenz & & $\begin{array}{l}\text { Alta calidad } \\
\text { (6 años) }\end{array}$ & Registro calificado \\
\hline Pontificia Universidad Javeriana & $\begin{array}{l}\text { Alta calidad } \\
\text { (8 años) }\end{array}$ & $\begin{array}{l}\text { Alta calidad } \\
\text { (Bogotá } 8 \text { años) } \\
\text { (Cali } 6 \text { años) }\end{array}$ & Registro calificado \\
\hline Universidad Católica de Colombia & $\begin{array}{l}\text { Alta calidad } \\
\text { (4 años })\end{array}$ & $\begin{array}{l}\text { Alta calidad } \\
\text { (8 años })\end{array}$ & Registro calificado \\
\hline Universidad Santo Tomás & $\begin{array}{l}\text { Alta calidad } \\
\text { (6 años) }\end{array}$ & $\begin{array}{c}\text { Alta calidad } \\
\text { (Bogotá } 6 \text { años) }\end{array}$ & Registro calificado \\
\hline Universidad Católica Luis Amigó-Funlam & & $\begin{array}{c}\text { Alta calidad } \\
\text { (Medellín } 6 \text { años) }\end{array}$ & Registro calificado \\
\hline
\end{tabular}

Nota. En negrilla se resalta la acreditación de alta calidad del programa de doctorado.

* Universidades del sector oficial. 
la Educación Superior cuenta con tres componentes relacionados entre sí —información, evaluación y fomentoademás del desarrollo profesional de los docentes, directivos y de los procesos de investigación.

Por último, sobresale que, dentro de los programas de doctorado aprobados, solo 2 programas - Universidad del Valle y Universidad del Norte- (el 15.4 \% de los programas) cuentan con el reconocimiento de alta calidad, y que, precisamente, se trata de los dos programas más antiguos de Colombia. Se esperaría que, con el transcurso del tiempo, los programas se vayan certificando en alta calidad.

\section{Indicadores en la producción académica de la psicología en Colombia}

Las publicaciones académicas realizadas en revistas especializadas representan por lo general contribuciones a su campo de experticia a través de hallazgos en materia de investigación o a partir de una recopilación de hallazgos presentada en un formato de revisión (Bajwa \& König, 2019; Harzing, 2008). De este modo, tradicionalmente se ha considerado que la cantidad de publicaciones está directamente relacionada con la visibilidad (Garfield, 1992; Liberatore \& Hermosilla, 2008). Este fenómeno de visibilización ha ocasionado un crecimiento acelerado en la productividad tanto a nivel nacional como internacional; productividad que incide directamente en casi todos los aspectos de la trayectoria de un investigador, incluyendo desde las decisiones de permanencia hasta las solicitudes de financiación (Abramo et al., 2011; Krampen, 2008).

En Colombia, la psicología, además de ser una profesión, es una disciplina con prácticas investigativas específicas que abarcan diversas líneas de investigación y áreas de experticia (López-López et al., 2018); aspecto que ha tenido un impacto importante en el incremento de la producción académica indexada por parte de las IES en las bases de datos de Scopus durante la última década (véase Figura 3).

Como se observa en la Figura 3, hay un crecimiento importante de la producción académica de la psicología en Colombia en los últimos años, con excepción de los años 2014 y 2015, en los cuales se presenta el menor registro de documentos indexados -el $21 \%$ de la producción académica emerge en estos dos años- A partir de esto, podemos afirmar que en la última década hay una tendencia clara de crecimiento, y que este es un acontecimiento que se acentúa en las dinámicas de investigación al interior de la disciplina en el país. En la Figura 4 se detalla este crecimiento, diferenciando la productividad en cada una de las instituciones de educación superior.

Como vemos, el porcentaje de crecimiento de la producción académica de la psicología colombiana representa un aumento del $204 \%$, pero la línea de citación presenta

Figura 3.

Frecuencia de la producción académica indexada en Scopus

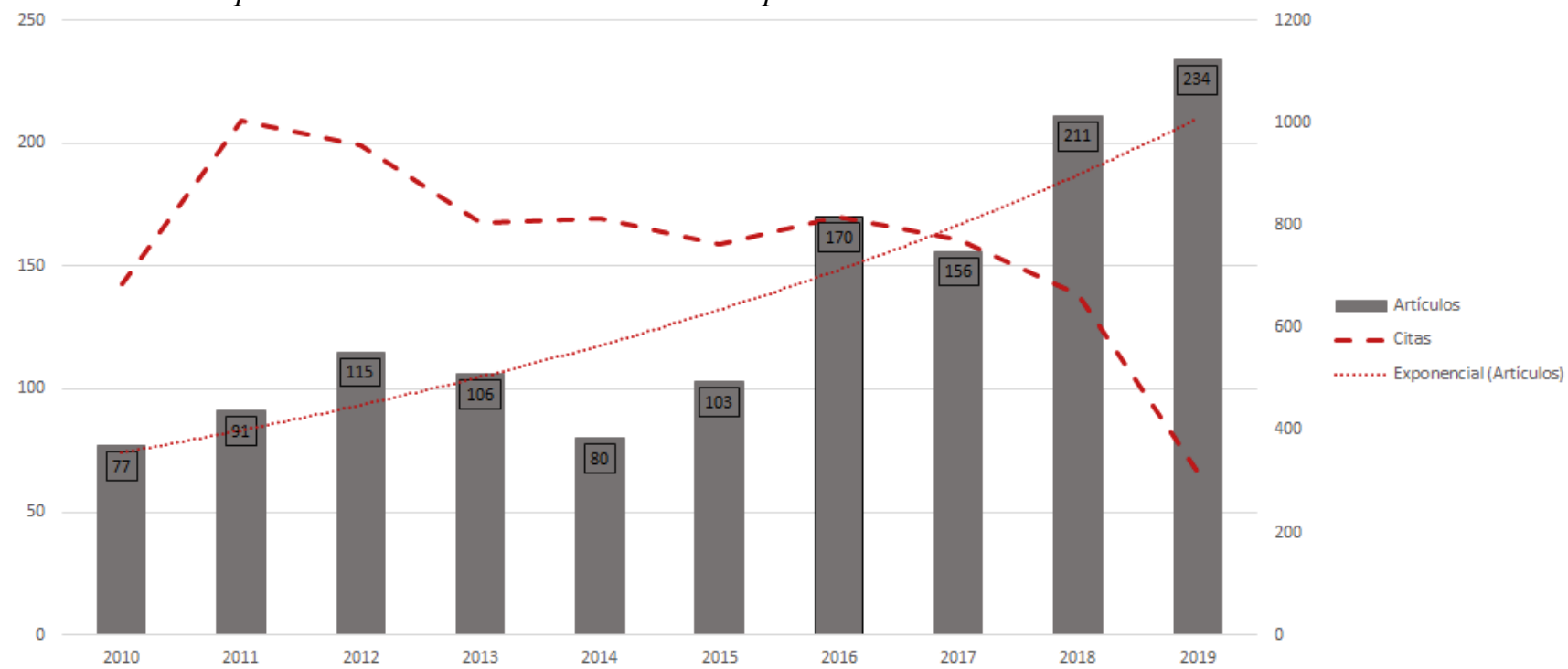

Fuente: elaboración propia. 
Figura 4.

Producción por año de los programas de doctorado

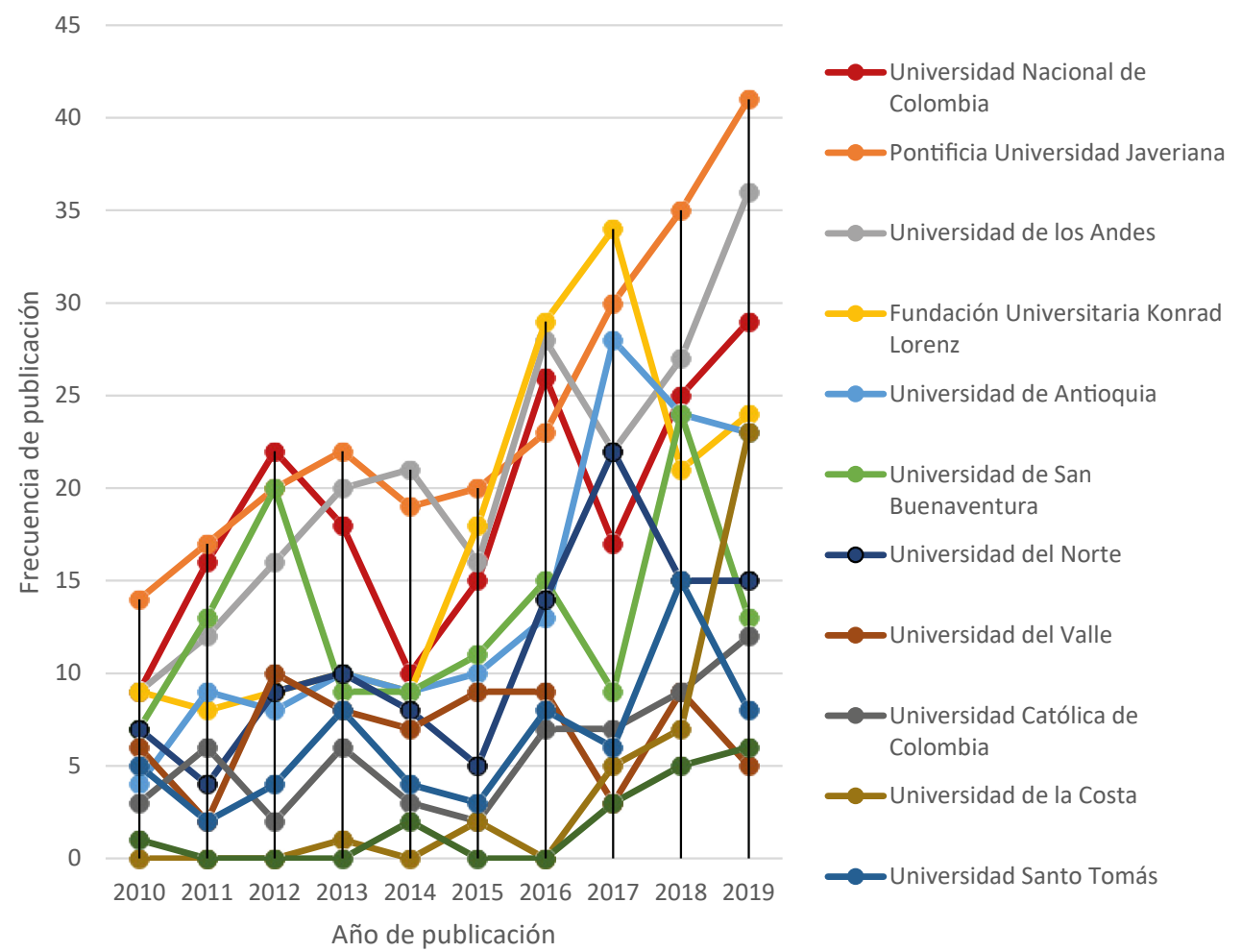

Nota. Fuente: elaboración propia.

un comportamiento dinámico, cuyo pico más alto se evidencia en el año 2011, cuando se superó las mil citas. En este periodo, el total de citas obtenidas al interior de esta producción fue de 7.82. Si bien es cierto que estos datos muestran un indicador general, es necesario detallar algunos aspectos. En la Tabla 3 se muestra el trabajo de las IES en función de la producción académica registrada, y su impacto en términos de citación.

La apuesta por visibilizar la investigación que se desarrolla al interior de la universidad colombiana en lo corrido de la última década puede entenderse como una tendencia a internacionalizar la investigación nacional. En este sentido, se ha visto que, aunque en términos generales la psicología en Colombia comienza a avanzar en materia de visibilidad e impacto (Salas et al., 2019), el número de documentos o citas son indicadores insuficientes de la productividad y visibilidad. Un indicador adicional de este espectro es el impacto, el cual, para la psicología en Colombia, requiere de la comunidad académica y de instituciones de educación para avanzar.
Tabla 3.

Producción académica e impacto de universidades con programas de doctorado en psicología

\begin{tabular}{lcccc}
\hline \multirow{2}{*}{ Universidad } & \multicolumn{3}{c}{ Artículos } & Citas \\
\cline { 2 - 5 } & $\mathrm{N}$ & $\%$ & $\mathrm{~N}$ & $\%$ \\
\hline Pontificia Universidad Javeriana & 240 & 18 & 1526 & 20.1 \\
Universidad de los Andes & 212 & 16 & 1462 & 19.3 \\
Universidad Nacional de Colombia & 188 & 14 & 496 & 6.5 \\
Fundación Universitaria & 171 & 13 & 700 & 9.2 \\
Konrad Lorenz & 138 & 10 & 1241 & 16.3 \\
Universidad de Antioquia & 135 & 10 & 1004 & 13.2 \\
Universidad de San Buenaventura & 109 & 8 & 707 & 9.3 \\
Universidad del Norte & 68 & 5 & 237 & 3.0 \\
Universidad del Valle & 58 & 4 & 143 & 1.9 \\
Universidad Católica de Colombia & 38 & 3 & 94 & 1.2 \\
Universidad de la Costa & 37 & 3 & 167 & 2.2 \\
Universidad Santo Tomás & 17 & 1 & 54 & 0.7 \\
Universidad Simón Bolívar & 1343 & 100 & 7826 & 100 \\
Total & & & &
\end{tabular}

Fuente: elaboración propia. 
En la Tabla 4 se muestra la frecuencia de autores que contribuyen con la producción académica de la psicología en Colombia, en función de las citas obtenidas.

Tabla 4.

Frecuencia de autores firmantes por documento

\begin{tabular}{cccc}
\hline $\mathrm{N}^{\circ}{ }^{\circ}$ autores & $\mathrm{N}^{\circ}{ }^{\circ}$ artículos & Citas & \% autores \\
\hline 1 & 159 & 197 & 11.8 \\
2 & 306 & 1155 & 22.8 \\
3 & 272 & 1236 & 20.3 \\
4 & 182 & 961 & 13.6 \\
5 & 120 & 559 & 8.9 \\
6 & 84 & 451 & 6.3 \\
7 & 54 & 399 & 4.0 \\
8 & 41 & 274 & 3.1 \\
9 & 22 & 188 & 1.6 \\
10 & 11 & 286 & 0.8 \\
11 & 21 & 267 & 1.6 \\
$12-15$ & 21 & 235 & 1.6 \\
$16-20$ & 18 & 387 & 1.3 \\
$20-30$ & 20 & 428 & 1.5 \\
$31-40$ & 6 & 470 & 0.4 \\
$41-60$ & 8 & 88 & 0.6 \\
$61-193$ & 6 & 192 & 0.4 \\
\hline
\end{tabular}

En este punto, es importante resaltar que el $94.7 \%$ $(n=1272)$ de la producción académica está en un rango de coautoría de uno a once autores por artículo. No obstante, la tendencia se ubica en el rango de uno a cinco autores, lo cual representa el $77 \%$ de los artículos registrados. Además, los datos muestran un $6 \%$ de artículos firmados por más de 20 autores; y que, por otra parte, no se evidencia fenómenos de hiperautoría, una estrategia que representa una inflación en los datos de las publicaciones y una práctica que cuestiona el mérito y las contribuciones dentro de los coautores (Cronin, 2001).

Al detallar las revistas donde se publicaron estas investigaciones, es posible dar cuenta de algunos elementos que representan parte del crecimiento de la psicología como disciplina, como lo es la importancia de las revistas nacionales como canal de comunicación científica en este proceso de internacionalización y visibilidad de la psicología colombiana (Cudina et al., 2017; Polanco-Carrasco et al., 2017; Polanco et al., 2017), pues el $35 \%(n=470)$ de la producción académica registrada se ha publicado en revistas nacionales. En la Figura 5 se presentan las revistas especializadas donde más publican los psicólogos en Colombia.

Asimismo, es importante resaltar que las revistas internacionales que publican y visibilizan la producción académica local se enmarcan en el crecimiento regional que ha tenido la disciplina (Romero-Torres et al., 2013; Russell et al., 2007). Este es el caso de importantes revistas de habla hispana como Terapia Psicológica, Revista Interamericana de Psicología, Revista Argentina de Clínica Psicológica, y revistas norteamericanas y europeas como Frontiers in Psychology, International Journal of Psychology and Psychological Therapy, y Physiology and Behavior. Estas revistas se constituyen como un canal de comunicación

Figura 5.

Top de 17 de revistas de mayor contribución a las publicaciones en psicología

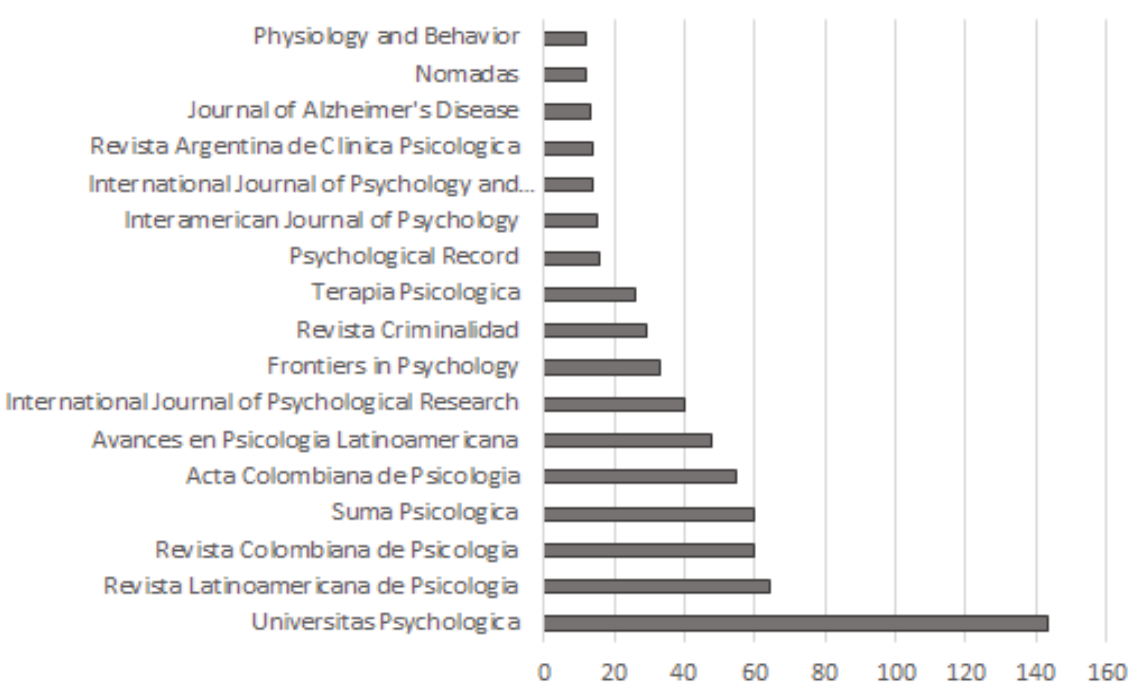

Nota. Fuente: elaboración propia. 
científica al interior de la psicología en el país, y es importante reconocer que algunas de estas son clasificadas en los primeros cuartiles en la clasificación de Scopus.

Productividad registrada en el Curriculum Vitae de Latinoamérica y el Caribe (CvLAC)

Uno de los objetivos del presente artículo fue determinar la relación entre la productividad en psicología en Colombia y la investigación de formación doctoral. Aunque no es posible tener un indicador directo, el registro de los artículos, libros y dirección en tesis de posgrado en psicología por parte de los investigadores asociados a los programas de doctorado de psicología responde parcialmente a este objetivo.

En este sentido, en total se recolectó la información de 193 profesores (véase Tabla 5) adscritos a las universidades que ofertan los programas de doctorado. A partir de ello, se contabilizaron los productos de artículos, distinguiendo entre revistas nacionales - colombianas - e internacionales, aunque no se realizó una clasificación de la indexación de dichas revistas. Sin embargo, contrastando con los resultados de la producción indizada en Scopus, es claro que las revistas nacionales e internacionales reportadas en este apartado difieren de la productividad encontrada en la base de datos de Scopus — que abarca más de 39000 revistas-. Esto sugiere que gran parte de esta productividad, al no encontrarse indizada, podría terminar siendo poco visible. En la Tabla 5 se presentan los resultados de los productos académicos registrados en el CvLAC de cada uno de los docentes incluidos en el estudio; y en la Tabla 6 se muestra un resumen de los registros de los productos asociados a las universidades de cada programa de doctorado en psicología registrados en el CvLAC.

Tabla 5.

Productividad en el CvLAC de los profesores asociados a los programas de psicología que ofrecen doctorado en psicología

\begin{tabular}{|c|c|c|c|c|c|c|}
\hline Nombre & Universidad & Rev. Nacional & Rev. Internacional & Total art. & Libros & Tesis posgrados \\
\hline Sandra Jimena Báez Buitrago & \multirow{18}{*}{$\begin{array}{l}\text { Universidad } \\
\text { de los Andes }\end{array}$} & 26 & 38 & 64 & 1 & 7 \\
\hline Carlos Andrés Gantiva Díaz & & 35 & 20 & 55 & 2 & 9 \\
\hline Luis Fernando Cárdenas Parra & & 21 & 25 & 46 & 0 & 20 \\
\hline Viviola Mélida Gómez Ortiz & & 29 & 16 & 45 & 2 & 25 \\
\hline Diana María Agudelo Vélez & & 19 & 20 & 39 & 3 & 19 \\
\hline Carolyn Finck Barboza & & 11 & 18 & 29 & 0 & 7 \\
\hline Enrique Chaux Torres & & 14 & 13 & 27 & 5 & 32 \\
\hline Elvia Vargas Trujillo & & 17 & 10 & 27 & 31 & 15 \\
\hline Florencia Reali Arcos & & 5 & 20 & 25 & 0 & 4 \\
\hline Yvonne Gómez Maquet & & 19 & 5 & 24 & 3 & 10 \\
\hline Karen Judith Ripoll Núñez & & 8 & 11 & 19 & 3 & 6 \\
\hline Julio Eduardo Cruz Vásquez & & 16 & 2 & 18 & 0 & 11 \\
\hline Sonia Carrillo Ávila & & 14 & 4 & 18 & 2 & 25 \\
\hline Amanda Milena Muñoz Martínez & & 7 & 11 & 18 & 2 & 13 \\
\hline Leonidas Castro Camacho & & 8 & 9 & 17 & 0 & 40 \\
\hline Juan Pablo Aranguren Romero & & 9 & 5 & 14 & 7 & 14 \\
\hline William Jiménez Leal & & 7 & 6 & 13 & 0 & 10 \\
\hline Ana Lucía Jaramillo Sierra & & 1 & 12 & 13 & 0 & 1 \\
\hline Mario Elkin Ramírez Ortiz & \multirow{8}{*}{$\begin{array}{l}\text { Universidad } \\
\text { de Antioquia }\end{array}$} & 32 & 25 & 57 & 15 & 15 \\
\hline Jesús Gallo & & 27 & 7 & 34 & 23 & 35 \\
\hline Juan Manuel Martin Uribe Cano & & 15 & 2 & 17 & 9 & 16 \\
\hline Angela María Jaramillo Burgos & & 12 & 0 & 12 & 8 & 4 \\
\hline Julio Eduardo Hoyos Zuluaga & & 10 & 1 & 11 & 1 & 14 \\
\hline Mauricio Fernández Arcila & & 10 & 0 & 10 & 5 & 6 \\
\hline Humberto Acosta Mesa & & 2 & 1 & 3 & 0 & 4 \\
\hline Carlos Calle Madrid & & 1 & 0 & 1 & 1 & 4 \\
\hline
\end{tabular}




\begin{tabular}{|c|c|c|c|c|c|c|}
\hline Nombre & Universidad & Rev. Nacional & Rev. Internacional & Total art. & Libros & Tesis posgrados \\
\hline Bertha Lucia Avendaño Prieto & \multirow{10}{*}{$\begin{array}{c}\text { Universidad } \\
\text { Católica de } \\
\text { Colombia }\end{array}$} & 31 & 4 & 35 & 0 & 35 \\
\hline María Idaly Barreto Galeano & & 26 & 7 & 33 & 1 & 27 \\
\hline María Constanza Aguilar Bustamante & & 20 & 7 & 27 & 3 & 3 \\
\hline Iván Felipe Medina Arboleda & & 20 & 5 & 25 & 1 & 10 \\
\hline Fredy Garay Garay & & 11 & 8 & 19 & 0 & 17 \\
\hline Alba Lucía Meneses Báez & & 16 & 2 & 18 & 2 & 38 \\
\hline Sonia Gisela Ríos Cruz & & 2 & 2 & 4 & 2 & 6 \\
\hline Martha Del Rosario Peña Sarmiento & & 3 & 1 & 4 & 0 & 1 \\
\hline Eliana Ivette Ortiz Garzón & & 1 & 2 & 3 & 0 & 2 \\
\hline Juan Camilo Carvajal Builes & & 2 & 0 & 2 & 0 & 1 \\
\hline Margel Alejandra Parra Fernández & \multirow{20}{*}{$\begin{array}{l}\text { Universidad } \\
\text { de la Costa }\end{array}$} & 5 & 15 & 20 & 2 & 4 \\
\hline Mónica Patricia Acuña Rodríguez & & 5 & 2 & 7 & & 3 \\
\hline Aura Alicia Cardozo Rusinque & & 1 & 5 & 6 & 6 & 10 \\
\hline Irmina Beatriz Hernández Sánchez & & 3 & 3 & 6 & 0 & 4 \\
\hline Adriana Angélica De La Peña Leiva & & 2 & 3 & 5 & 3 & 5 \\
\hline Diana Carolina Pérez Pedraza & & 1 & 4 & 5 & 2 & 0 \\
\hline María Jesús Ramírez Ordoñez & & 1 & 3 & 4 & 0 & 0 \\
\hline Claudia Patricia Arenas Rivera & & 0 & 3 & 3 & 0 & 4 \\
\hline Jholman Luis García Padilla & & 1 & 2 & 3 & 0 & 3 \\
\hline Luz Miriam Ibáñez Navarro & & 2 & 1 & 3 & 0 & 0 \\
\hline Marcela De La Hoz Álvarez & & 0 & 2 & 2 & 0 & 0 \\
\hline Judys Alfaro Álvarez & & 1 & 1 & 2 & 0 & 0 \\
\hline Alex José Vásquez Mercado & & 0 & 1 & 1 & 0 & 2 \\
\hline Karen Elena Salas Viloria & & 1 & 0 & 1 & 0 & 0 \\
\hline Gina Isabel Colmenares López & & 1 & 0 & 1 & 2 & 0 \\
\hline Aldair Morales Cuadro & & 0 & 1 & 1 & 0 & 0 \\
\hline Marlis Isabel Cardona Villa & & 0 & 0 & 0 & 0 & 0 \\
\hline Eloy Huamani Ferrer & & 0 & 0 & 0 & 0 & 0 \\
\hline Elizabeth Diazgranados Sotomayor & & 0 & 0 & 0 & 0 & 0 \\
\hline Briguette Chegwin Díaz & & 0 & 0 & 0 & 0 & 0 \\
\hline Wilson López-López & \multirow{18}{*}{$\begin{array}{l}\text { Pontificia } \\
\text { Universidad } \\
\text { Javeriana }\end{array}$} & 83 & 59 & 142 & 9 & 4 \\
\hline Hernán Camilo Pulido Martínez & & 16 & 10 & 26 & 2 & 4 \\
\hline Gloria Patricia Marciales Vivas & & 15 & 6 & 21 & 6 & 1 \\
\hline Olga Alicia Carbonell Blanco & & 6 & 12 & 18 & 2 & 4 \\
\hline Luis Manuel Silva Martín & & 12 & 5 & 17 & 0 & 0 \\
\hline Milton Eduardo Bermúdez Jaimes & & 12 & 2 & 14 & 4 & 2 \\
\hline Leonardo Alberto Rodríguez Cely & & 10 & 3 & 13 & 4 & 11 \\
\hline Mario Fernando Gutiérrez Romero & & 6 & 7 & 13 & 1 & 1 \\
\hline María Margarita Echeverry Buriticá & & 3 & 7 & 10 & 2 & 7 \\
\hline Claudia Constanza Tovar Guerra & & 7 & 3 & 10 & 0 & 1 \\
\hline Johanna Burbano Valente & & 6 & 0 & 6 & 2 & 1 \\
\hline Hernando Taborda Osorio & & 2 & 3 & 5 & 0 & 1 \\
\hline Rocío Abello Correa & & 4 & 0 & 4 & 4 & 1 \\
\hline Olga Lucia Huertas Hernández & & 4 & 0 & 4 & 0 & 0 \\
\hline Alba Luz Giraldo Tamayo & & 2 & 0 & 2 & 3 & 1 \\
\hline Jaime Collazos Aldana & & 2 & 0 & 2 & 2 & 3 \\
\hline Irene Giovanni Aguilar & & 0 & 1 & 1 & 1 & 7 \\
\hline Claudia Carolina Botero García & & 1 & 0 & 1 & 0 & 6 \\
\hline
\end{tabular}


Producción de los doctorados en psicología en Colombia

\begin{tabular}{|c|c|c|c|c|c|c|}
\hline Nombre & Universidad & Rev. Nacional & Rev. Internacional & Total art. & Libros & Tesis posgrados \\
\hline María Teresa Varela Arévalo & \multirow{12}{*}{$\begin{array}{c}\text { Pontificia } \\
\text { Universidad } \\
\text { Javeriana, Cali }\end{array}$} & 30 & 11 & 41 & 3 & 5 \\
\hline Solanlly Ochoa Angrino & & 13 & 3 & 16 & 2 & 4 \\
\hline Natalia Cadavid Ruiz & & 8 & 7 & 15 & 0 & 4 \\
\hline Victoria Eugenia Acevedo Velasco & & 9 & 6 & 15 & 6 & 6 \\
\hline Teresita María Sevilla Peñuela & & 12 & 1 & 13 & 2 & 6 \\
\hline María Irene Victoria Morales & & 9 & 3 & 12 & 2 & 3 \\
\hline Tatiana Rojas Ospina & & 6 & 5 & 11 & 3 & 5 \\
\hline Fabián Bravo Reyes & & 1 & 3 & 4 & 0 & 2 \\
\hline James Cuenca Morales & & 2 & 1 & 3 & 1 & 10 \\
\hline Martha Patricia Romero Caraballo & & 2 & 1 & 3 & 0 & 3 \\
\hline Jimena Botero Sarassa & & 1 & 1 & 2 & 1 & 1 \\
\hline Ivonne Leadith Díaz Pérez & & 2 & 0 & 2 & 17 & 0 \\
\hline Francisco José Ruiz & \multirow{9}{*}{$\begin{array}{c}\text { Fundación } \\
\text { Universitaria } \\
\text { Konrad Lorenz }\end{array}$} & 14 & 60 & 74 & 0 & 14 \\
\hline Mónica María Novoa & & 47 & 12 & 59 & 0 & 41 \\
\hline Pablo Vallejo Medina & & 6 & 45 & 51 & 1 & 13 \\
\hline Camilo Hurtado Parrado & & 18 & 25 & 43 & 0 & 4 \\
\hline Marithza Sandoval & & 33 & 4 & 37 & 2 & 34 \\
\hline Leonardo Augusto Ortega Murillo & & 9 & 18 & 27 & 0 & 1 \\
\hline Gustavo René García Vargas & & 4 & 16 & 20 & 0 & 5 \\
\hline Javier Leonardo Rico Rodríguez & & 4 & 12 & 16 & 0 & 0 \\
\hline Myriam Carmenza Sierra Puentes & & 4 & 4 & 8 & 1 & 13 \\
\hline José Ignacio Ruiz Pérez & \multirow{26}{*}{$\begin{array}{c}\text { Universidad } \\
\text { Nacional de } \\
\text { Colombia }\end{array}$} & 87 & 8 & 95 & 5 & 2 \\
\hline Germán Antonio Gutiérrez Domínguez & & 36 & 13 & 49 & 5 & 15 \\
\hline Jaime Yáñez Canal & & 43 & 3 & 46 & 8 & 22 \\
\hline María Patricia Montañés Ríos & & 33 & 8 & 41 & 6 & 2 \\
\hline Marisol Lamprea Rodríguez & & 17 & 16 & 33 & 0 & 24 \\
\hline Javier Alejandro Corredor Aristizabal & & 17 & 12 & 29 & 2 & 13 \\
\hline María Elvia Domínguez Blanco & & 23 & 2 & 25 & 8 & 16 \\
\hline Aura Nidia Herrera Rojas & & 16 & 5 & 21 & 0 & 18 \\
\hline Ricardo Tamayo Osorio & & 11 & 8 & 19 & 0 & 3 \\
\hline José Gregorio Rodríguez & & 14 & 4 & 18 & 5 & 1 \\
\hline Carlos Parales Quenza & & 11 & 6 & 17 & 2 & 3 \\
\hline María Clemencia Castro Vergara & & 15 & 0 & 15 & 10 & 0 \\
\hline Carmen Elvira Navia Arroyo & & 10 & 4 & 14 & 6 & 11 \\
\hline Olga Rosalba Rodríguez Jiménez & & 13 & 1 & 14 & 0 & 7 \\
\hline Juan De Jesús Guerrero Guerrero & & 12 & 0 & 12 & 0 & 14 \\
\hline Magnolia Del Pilar Ballesteros Cabrera & & 7 & 4 & 11 & 0 & 21 \\
\hline Álvaro Arturo Clavijo Álvarez & & 10 & 0 & 10 & 2 & 8 \\
\hline Eduardo Aguirre Dávila & & 6 & 3 & 9 & 15 & 25 \\
\hline Pablo Muñoz Specht & & 0 & 7 & 7 & 0 & 4 \\
\hline Roberto Posada Gilède & & 3 & 3 & 6 & 0 & 6 \\
\hline Yalile Sánchez Hurtado & & 5 & 0 & 5 & 0 & 1 \\
\hline Carlos Alberto Acosta Riaño & & 4 & 0 & 4 & 2 & 1 \\
\hline Jazmine Escobar Pérez & & 4 & 0 & 4 & 0 & 1 \\
\hline Oscar Mauricio Gallego Villa & & 3 & 0 & 3 & 0 & 0 \\
\hline Luz Amparo Pérez Fonseca & & 3 & 0 & 3 & 0 & 2 \\
\hline Emilio Meluk Castro & & 0 & 0 & 0 & 1 & 0 \\
\hline
\end{tabular}




\begin{tabular}{|c|c|c|c|c|c|c|}
\hline Nombre & Universidad & Rev. Nacional & Rev. Internacional & Total art. & Libros & Tesis posgrados \\
\hline Jorge Enrique Palacio Sañudo & \multirow{25}{*}{$\begin{array}{l}\text { Universidad } \\
\text { del Norte }\end{array}$} & 64 & 29 & 93 & 12 & 72 \\
\hline José Juan Amar Amar & & 80 & 12 & 92 & 50 & 42 \\
\hline Raimundo Abello Llanos & & 61 & 5 & 66 & 17 & 38 \\
\hline Camilo Alberto Madariaga Orozco & & 47 & 9 & 56 & 11 & 46 \\
\hline Rafael De Jesús Tuesca Molina & & 35 & 20 & 55 & 12 & 42 \\
\hline Alberto Mario De Castro Correa & & 30 & 8 & 38 & 8 & 13 \\
\hline María Del Carmen Amarís Macías & & 31 & 3 & 34 & 1 & 67 \\
\hline Jesús Antonio Arroyave Cabrera & & 13 & 15 & 28 & 6 & 16 \\
\hline Jean David Polo Vargas & & 19 & 8 & 27 & 0 & 20 \\
\hline Carlos José De Los Reyes Aragón & & 9 & 17 & 26 & 1 & 16 \\
\hline Ana María Trejos Herrera & & 11 & 13 & 24 & 8 & 9 \\
\hline Olga Lucía Hoyos De Los Ríos & & 19 & 3 & 22 & 1 & 30 \\
\hline Ana Rita Russo De Vivo & & 16 & 6 & 22 & 90 & 15 \\
\hline Moisés Roberto Mebarak Chams & & 13 & 6 & 19 & 0 & 9 \\
\hline Carlos Alberto Acosta Barros & & 16 & 0 & 16 & 0 & 29 \\
\hline José Alfredo Aparicio Serrano & & 9 & 5 & 14 & 1 & 9 \\
\hline $\begin{array}{l}\text { Edith Teresa María Aristizabal Díaz- } \\
\text { Granados }\end{array}$ & & 12 & 1 & 13 & 1 & 3 \\
\hline Marina Llanos Martínez & & 13 & 0 & 13 & 5 & 5 \\
\hline Miguel Rojas Santiago & & 4 & 8 & 12 & 1 & 8 \\
\hline Mayilin De Los Santos Moreno Torres & & 9 & 2 & 11 & 1 & 9 \\
\hline Soraya Lewis Harb & & 9 & 2 & 11 & 1 & 14 \\
\hline María Mercedes Botero Posada & & 8 & 1 & 9 & 2 & 4 \\
\hline Isabel Cristina Suarez Del Chiaro & & 1 & 6 & 7 & 0 & 2 \\
\hline Ana Mercedes Bello Villanueva & & 4 & 2 & 6 & 0 & 43 \\
\hline Tania Jannett Iglesias Rodríguez & & 1 & 4 & 5 & 1 & 3 \\
\hline Daniel Camilo Aguirre Acevedo & \multirow{12}{*}{$\begin{array}{l}\text { Universidad } \\
\text { de San } \\
\text { Buenaventura }\end{array}$} & 56 & 46 & 102 & 0 & 29 \\
\hline Henry Castillo Parra & & 10 & 3 & 13 & 0 & 10 \\
\hline Jorge Mauricio Cuartas Arias & & 27 & 10 & 37 & 0 & 32 \\
\hline Ana Milena Gaviria Gómez & & 17 & 32 & 49 & 0 & 13 \\
\hline Nora Helena Londoño Arredondo & & 38 & 4 & 42 & 5 & 24 \\
\hline Juan Pablo Lopera Vásquez & & 8 & 9 & 17 & 0 & 2 \\
\hline David Andrés Montoya Arenas & & 26 & 2 & 28 & 1 & 32 \\
\hline Norman Darío Moreno Carmona & & 16 & 5 & 21 & 7 & 9 \\
\hline David Antonio Pineda Salazar & & 53 & 86 & 139 & 6 & 51 \\
\hline Carlos Arturo Sandoval Casilimas & & 9 & 1 & 10 & 15 & 38 \\
\hline Carla María Zapata Rueda & & 6 & 4 & 10 & 2 & 14 \\
\hline Maryoris Elena Zapata Zabala & & 15 & 2 & 17 & 2 & 37 \\
\hline Josep María Blanch Ribas & \multirow{5}{*}{$\begin{array}{c}\text { Universidad } \\
\text { de San } \\
\text { Buenaventura, } \\
\text { Cali }\end{array}$} & 7 & 38 & 45 & 34 & 28 \\
\hline Diana Britto Ruiz & & 10 & 0 & 10 & 14 & 0 \\
\hline Silvia Cristina Caicedo Muñoz & & 6 & 3 & 9 & 2 & 42 \\
\hline Wilner Arbey Riascos Sánchez & & 1 & 1 & 2 & 2 & 3 \\
\hline Carlos Andrés Sánchez Jaramillo & & 1 & 1 & 2 & 0 & 1 \\
\hline
\end{tabular}




\begin{tabular}{|c|c|c|c|c|c|c|}
\hline Nombre & Universidad & Rev. Nacional & Rev. Internacional & Total art. & Libros & Tesis posgrados \\
\hline Teresita De Lourdes Bernal Romero & \multirow{8}{*}{$\begin{array}{l}\text { Universidad } \\
\text { Santo Tomás }\end{array}$} & 18 & 5 & 23 & 2 & 30 \\
\hline Ricardo Arturo Jaramillo Moreno & & 11 & 1 & 12 & 1 & 8 \\
\hline $\begin{array}{l}\text { Claudia Ligia Esperanza Charry } \\
\text { Poveda }\end{array}$ & & 0 & 8 & 8 & 0 & 1 \\
\hline Luz Amparo Serrano Quintero & & 4 & 3 & 7 & 8 & 2 \\
\hline Melba Ximena Figueroa Ángel & & 5 & 1 & 6 & 0 & 0 \\
\hline Ericka Giovanna Mayorga Sierra & & 5 & 1 & 6 & 0 & 1 \\
\hline Rosa Elena Duque García & & 2 & 0 & 2 & 2 & 47 \\
\hline Angie Paola Román Cárdenas & & 0 & 0 & 0 & 5 & 6 \\
\hline Nelson Molina Valencia & \multirow{22}{*}{$\begin{array}{c}\text { Universidad } \\
\text { del Valle }\end{array}$} & 20 & 14 & 34 & 10 & 15 \\
\hline Juan Felipe Cardona & & 0 & 27 & 27 & 0 & 11 \\
\hline Erico Rentería Pérez & & 18 & 9 & 27 & 12 & 41 \\
\hline Olga Lucia Obando Salazar & & 17 & 5 & 22 & 6 & 6 \\
\hline María Eugenia Delgado Gallego & & 8 & 11 & 19 & 2 & 3 \\
\hline Joseph Anthony Sampson & & 13 & 1 & 14 & 0 & 10 \\
\hline Gabriel Arteaga & & 10 & 1 & 11 & 0 & 8 \\
\hline María Cristina Tenorio Sampson & & 11 & 0 & 11 & 5 & 26 \\
\hline Yenny Otálora Sevilla & & 4 & 5 & 9 & 7 & 3 \\
\hline Álvaro Enríquez Martínez & & 8 & 1 & 9 & 2 & 9 \\
\hline María Eugenia Villalobos Valencia & & 6 & 2 & 8 & 4 & 12 \\
\hline Marlenny Guevara Guerrero & & 5 & 3 & 8 & 6 & 4 \\
\hline Oscar Aurelio Ordoñez Morales & & 5 & 2 & 7 & 2 & 12 \\
\hline Diana Patricia De Castro Daza & & 5 & 1 & 6 & 1 & 7 \\
\hline Diego Fernando Guerrero López & & 4 & 2 & 6 & 0 & 4 \\
\hline Hernán Sánchez Ríos & & 5 & 1 & 6 & 4 & 7 \\
\hline Oscar Martín Rosero Sarasty & & 5 & 1 & 6 & 1 & 3 \\
\hline William López Gutiérrez & & 4 & 2 & 6 & 0 & 0 \\
\hline Fátima Díaz Bambula & & 3 & 1 & 4 & 1 & 6 \\
\hline Rita Patricia Ocampo Cepeda & & 1 & 2 & 3 & 0 & 2 \\
\hline Eliana Isabel Bedoya Durán & & 1 & 0 & 1 & 0 & 0 \\
\hline María Angélica García López & & 0 & 0 & 0 & 0 & 10 \\
\hline
\end{tabular}

Nota. Tabla ordenada por cantidad total de artículos. No se tomaron los datos de profesores de la Universidad Simón Bolívar, al no contar con la información en la página ni del programa ni del doctorado. Promedio de artículos por universidad a nivel nacional (Rev. nacional) o internacional (Rev. internacional), suma total de los artículos.

Con esta información, se realizó una matriz de correlación entre los artículos publicados - nacionales e internacionaleslos libros publicados y las tesis de posgrado. El objetivo de este análisis fue determinar si la asesoría/dirección de tesis de posgrado tenía una relación con la productividad en las modalidades de artículos y libros. Esta relación también contó con las tesis de otros posgrados adicionales a las de doctorado.

Este análisis permite identificar si la producción de posgrados en psicología, además del doctorado, termina relacionándose con la cantidad de productos publicados. Como resultado, en la Figura 6a se observan los coeficientes de correlación de Spearman, en los que se encuentra que la mayor correlación se da entre la asesoría/dirección de tesis de posgrado y la publicación de artículos en revistas nacionales $(r h o=0.61 ; p<.05)$. Luego, aparece una correlación menor entre la asesoría/dirección de tesis de posgrado y los artículos en revistas internacionales ( $r h o=0.41 ; p<.05)$; $\mathrm{y}$, finalmente, una correlación menor con las tesis y los libros $(r h o=0.31 ; p<.05)$. Esos datos sugieren que la investigación realizada en los posgrados tiene una mayor probabilidad de haberse publicado en revistas nacionales que internacionales, y mucho menos en libros. La dispersión de los datos de estas comparaciones aparece en la Figura 6b-6d, donde se observa que en la relación entre artículos nacionales y tesis, la dispersión de los puntos sugiere una relación lineal que no se observa en las otras correlaciones. 
Tabla 6.

Consolidado de la producción académica en el CvLAC por universidades

\begin{tabular}{|c|c|c|c|c|c|c|c|c|c|}
\hline \multirow{2}{*}{ Universidad } & \multirow{2}{*}{ Profesores } & \multicolumn{2}{|c|}{ Rev. nacional } & \multicolumn{2}{|c|}{ Rev. internacional } & \multicolumn{2}{|c|}{ Total artículos } & \multirow{2}{*}{ Libros } & \multirow{2}{*}{$\begin{array}{c}\text { Tesis } \\
\text { posgrados }\end{array}$} \\
\hline & & Promedio & Total & Promedio & Total & Promedio & Total & & \\
\hline Universidad del Norte* & 25 & 21.36 & 534 & 7.4 & 185 & 28.76 & 719 & 230 & 564 \\
\hline Universidad de los Andes & 18 & 14.77 & 266 & 13.61 & 245 & 28.38 & 511 & 61 & 268 \\
\hline Universidad Nacional de Colombia* & 26 & 15.5 & 403 & 4.11 & 107 & 19.61 & 510 & 77 & 220 \\
\hline Universidad de San Buenaventura, Medellín & 12 & 23.41 & 281 & 17 & 204 & 40.41 & 485 & 38 & 291 \\
\hline Fundación Universitaria Konrad Lorenz & 9 & 15.44 & 139 & 21.77 & 196 & 37.22 & 335 & 4 & 125 \\
\hline Pontificia Universidad Javeriana & 18 & 10.61 & 191 & 6.55 & 118 & 17.16 & 309 & 42 & 55 \\
\hline Universidad del Valle* & 22 & 6.95 & 153 & 4.13 & 91 & 11.09 & 244 & 63 & 199 \\
\hline Universidad Católica de Colombia & 10 & 13.2 & 132 & 3.8 & 38 & 17 & 170 & 9 & 140 \\
\hline Universidad de Antioquia & 8 & 13.62 & 109 & 4.5 & 36 & 18.12 & 145 & 62 & 98 \\
\hline Pontificia Universidad Javeriana, Cali & 12 & 7.91 & 95 & 3.5 & 42 & 11.41 & 137 & 37 & 49 \\
\hline Universidad Santo Tomás & 9 & 7.22 & 65 & 2.88 & 26 & 10.11 & 91 & 21 & 98 \\
\hline Universidad de la Costa* & 20 & 1.2 & 24 & 2.3 & 46 & 3.5 & 70 & 15 & 35 \\
\hline Universidad de San Buenaventura, Cali & 5 & 5 & 25 & 8.6 & 43 & 13.6 & 68 & 52 & 74 \\
\hline
\end{tabular}

Nota. Tabla ordenada por cantidad total de artículos. No se tomaron los datos de profesores de la Universidad Simón Bolívar, al no contar con la información en la página ni del programa ni del doctorado. Promedio de artículos por universidad a nivel nacional (Rev. nacional) o internacional (Rev. internacional), suma total de los artículos. * Universidades que no indicaban los profesores que dictaban en el doctorado y por tanto fueron incluidos todos los profesores asociados al programa de psicología.

Hay algunos datos que sobresalen en el análisis de estas relaciones, por ejemplo, la baja cantidad de tesis de posgrados y un elevado número de publicaciones internacionales, lo cual sería un indicador adicional de que la investigación realizada en los posgrados en psicología no es la que alimenta las publicaciones en revistas internacionales. Casos aislados de alta producción de libros y pocos artículos también sugieren que el número de libros publicados no relaciona con la investigación directamente; $y$ casos de un gran número de tesis y poco número de artículos internacionales indica una baja difusión de la investigación realizada en los posgrados.

Cuando se comparan los datos registrados en el CvLAC y la cantidad de producción en Scopus, se encuentra una gran diferencia entre los reportes. Si bien cada una obedece a fuentes diferentes, llama la atención que la producción reportada por los investigadores tiene como fuente principal las revistas nacionales, y al diferir de los datos de Scopus, se deriva que no son revistas indexadas en sistemas internacionales. Esta diferencia sugiere, además, que esta mayor publicación en revistas nacionales no indizadas internacionalmente tendría una baja visibilidad, y que no son publicadas en revistas consideradas de alta calidad e impacto, lo que resulta en un camino poco ideal a la hora de difundir conocimiento derivado de procesos de investigación. En este mismo sentido, el impacto de esta producción no puede ser establecido al no tener métricas de impacto y visibilidad de estas revistas que no se encuentran en dichos sistemas de indexación.

La otra posibilidad para explicar esta diferencia se centra en la forma como ha sido abordado el fenómeno de investigación/publicación por parte de estas instituciones. Dado que la cantidad de trabajos de posgrado en las instituciones supera la cantidad de artículos totales, se infiere que no se ha establecido una cultura de investigación-publicación en los posgrados y que, por el contrario, existe una gran cantidad de investigaciones que no terminan siendo difundidas o, que son difundidas en revistas locales o regionales, lo cual genera un efecto de poca visibilidad en ambos casos.

Finalmente, es importante señalar que los datos recolectados y analizados pueden presentar dos dificultades importantes. En primer lugar, la producción analizada es la registrada en el CvLAC personal y no se asocia directamente con las instituciones, esto significa que, si una persona cambia de afiliación institucional, su productividad cambiará de institución; caso contrario a lo que sucede en Scopus, donde 
Figura 6.

Relación entre la publicación de productos (artículos y libros) y la asesoría/dirección de tesis de posgrado
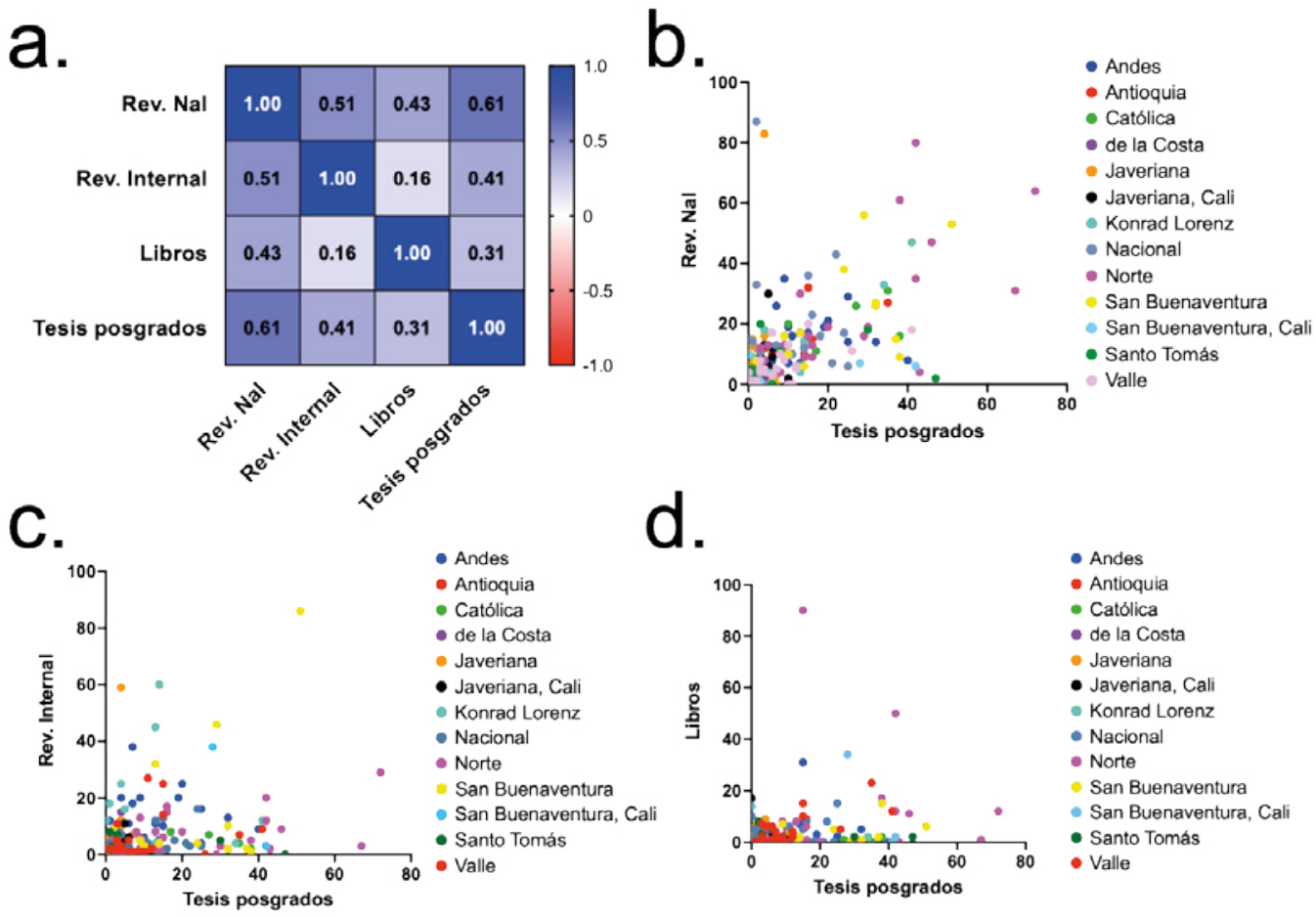

Nota. Fuente: elaboración propia.

se encuentra una productividad asociada más a las instituciones de afiliación. Esto es importante en algunos casos donde profesores de alto nivel de publicación cambiaron recientemente de filiación y ahora figuran en la institución actual, pero en Scopus su productividad esta asociada a la última institución firmante en los artículos. La segunda dificultad para resaltar es la trayectoria personal y académica de los autores, dado que, en la información que se extrajo del curriculum vitae de los profesores, aquellos con mayor experiencia registrarán más productos que no necesariamente estarán asociados a los doctorados. Aunque esta condición podría inflar parcialmente los registros de artículos, es claro que el aumento en la producción en los últimos 20 años ha crecido junto con la oferta de programas de posgrado, lo que sugiere que la producción más reciente es mayor, y que esta se daría en la ventana temporal que nos interesa.

Finalmente, adicional al análisis de productividad individual y de instituciones, otros factores han contribuido a explicar un aumento en esta producción, como lo son, por ejemplo, las redes académicas y de cooperación, que se presentan a continuación.
Redes de comunicación científica de la investigación en psicología en Colombia

Un aspecto central para potenciar el desarrollo y avance de la investigación depende del esfuerzo que se establece en los procesos de colaboración e internacionalización de la ciencia (Ávila-Toscano et al., 2019; Krabel et al., 2012; Russell et al., 2007). La colaboración dentro de las dinámicas de investigación ha sido una constante, particularmente en las universidades, las cuales tienen el papel y la misión de difundir conocimientos. La colaboración se sostiene como algo que debe fomentarse y, como hemos visto, a lo largo de los años ha habido una tendencia hacia su aumento (Jonkers \& Tijssen, 2008).

Los procesos de colaboración involucran el desarrollo de actividades de investigación conjuntas entre académicos de diversas afiliaciones institucionales y de diferentes países con un objetivo de investigación común, lo que proporciona beneficios mutuos (Carlsson, 2006). Específicamente, la colaboración se logra mediante acuerdos formales, firmados o tácitos, que son acordados por políticas nacionales para procesos de evaluación científica (Glänzel, 2001; Godin \& 
Gingras, 2000); a través de comunidades académicas mediante el intercambio de hallazgos y transferencias de conocimiento (Frenken, 2002). Algunos estudios han intentado identificar posibles intereses en común entre los investigadores para facilitar estos procesos de colaboración (Garcia et al., 2015).

En este sentido, la colaboración se posiciona como un recurso eficaz para la generación de nuevo conocimiento, especialmente para países y comunidades académicas que presentan índices de baja productividad científica y recursos limitados (Abdill et al., 2020). Este es el panorama que se ha presentado en Colombia, y, en el caso de la psicología, las redes de colaboración científica constituyen un fenómeno reciente, dado que en las últimas décadas hemos contado con los recursos que nos permitan llevar a cabo los análisis de redes sociales al interior de esta disciplina en el país (Armayones Ruiz et al., 2015; Ávila-Toscano et al., 2013; Ossa \& Cudina, 2016). Así pues, este trabajo colaborativo ha sido central para promover la investigación en red. Teniendo esto en cuenta, en la Figura 7 se muestran las redes de colaboración que se desprenden de las IES en Colombia que ofertan programas académicos de doctorados en psicología del país.

Figura 7.

Redes de colaboración de IES en Colombia

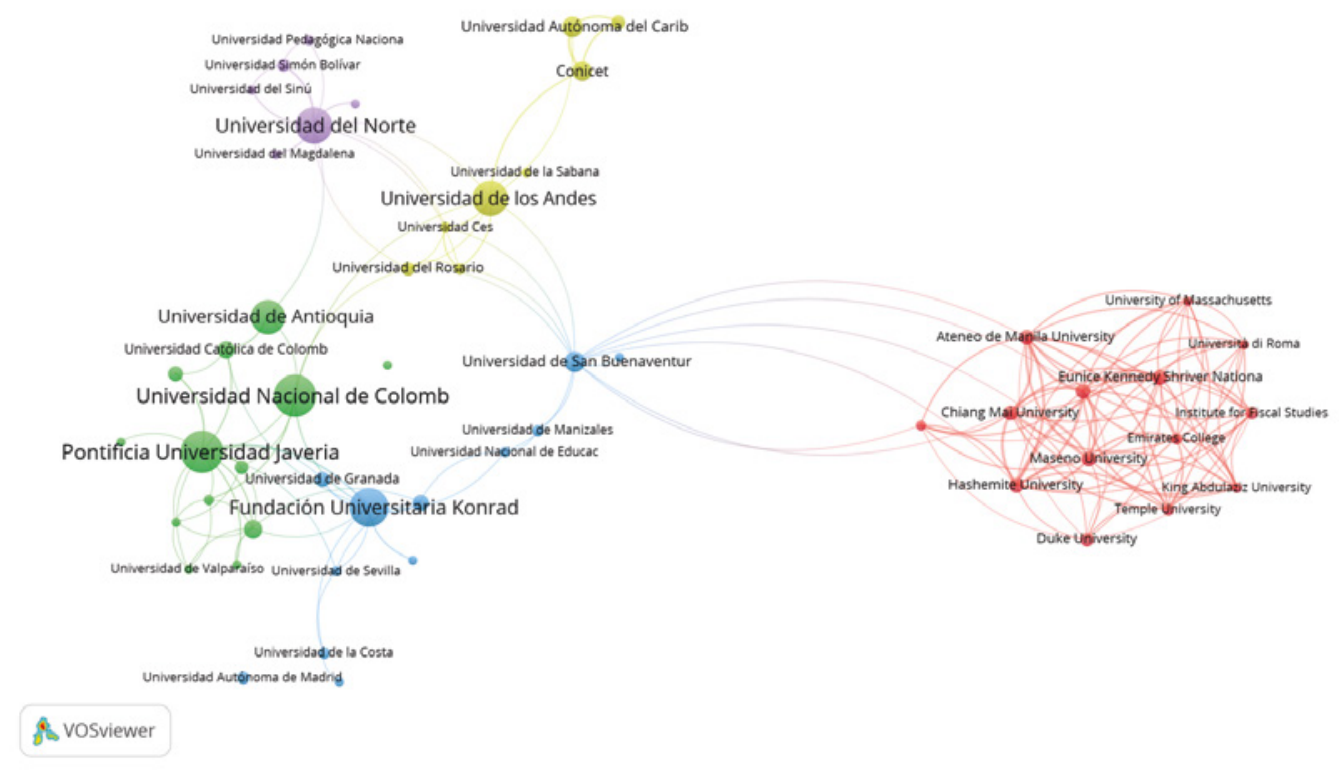

Nota. Fuente: elaboración propia.

Esta figura nos permite decantar la conformación de redes de colaboración entre instituciones de educación superior a nivel nacional e internacional. En particular, a nivel nacional cabe resaltar el trabajo colaborativo que se ha establecido a través de la Pontificia Universidad Javeriana, junto con la Fundación Universitaria Konrad Lorenz, la Universidad Nacional de Colombia, la Universidad del Norte, la Universidad de los Andes y la Universidad de San Buenaventura. De hecho, esta última ha servido de puente para estrechar los lazos de colaboración con otras instituciones del ámbito internacional, como lo son la Universidad de Massachusetts y la Universidad de Duke. Por otro lado, en la Figura 8 se muestran las redes de coautoría que se vienen configurando al interior de la disciplina por países para identificar un indicador de colaboración internacional, $y$, en cuanto a la autoría en colaboración, en la Tabla 7 se presentan los investigadores en psicología en Colombia que más promueven el trabajo colaborativo, a partir de los datos de coautoría.

Como se ha dicho, las dinámicas de investigación en psicología en el país permiten evidenciar el fortalecimiento de una comunidad académica a través de la colaboración nacional e internacional en diversas líneas y campos de investigación; aspecto que permite reafirmar la apuesta 
por visibilizar e internacionalizar la investigación local. De hecho, como se observa en la Figura 8, se encuentran hasta 50 países que constituyen las redes de colaboración en la investigación psicológica en Colombia; de estos, la mayor concentración de redes de colaboración se establece en Latinoamérica, con el $60.1 \%(n=1871)$, seguida de Europa, con el $20.6 \%(n=632)$, y Norte América, con el $9.4 \%(n=290)$ de la producción académica reportada.
El resto de la producción académica se distribuye en Asia, con $5.4 \%(n=166)$; Oceanía, con $2.5 \%(n=290)$; y África, con $1.2 \%(n=37)$. Claramente, la investigación de la psicología colombiana representa una parte cada vez más importante de la producción de conocimiento a nivel mundial. Sumado a esto, en la Tabla 8 se presentan los diez países que más contribuyen con la investigación nacional.

Tabla 7.

Autores colombianos con mayores relaciones de coautorías

\begin{tabular}{lcccc}
\hline \multicolumn{1}{c}{ Autor } & Enlaces & Total enlaces* & Documentos & Citaciones \\
\hline Lopera, F. & 28 & 104 & 44 & 987 \\
Uribe Tirado, L. M. & 26 & 159 & 82 & 302 \\
Bocanegra, Y. & 19 & 45 & 16 & 169 \\
García, A. M. & 17 & 73 & 41 & 336 \\
López-López, W. & 17 & 40 & 13 & 305 \\
Pineda, D. & 16 & 50 & 12 & 483 \\
Trujillo, N. & 16 & 50 & 7 & 241 \\
Herrera, E. & 15 & 29 & 16 & 96 \\
Pineda, D. A. & 15 & 43 & & 189 \\
\hline
\end{tabular}

Nota. * El total de enlaces se refiere a la fuerza de todas las relaciones de coautoría que se establece entre un investigador determinado con otros investigadores. Fuente: elaboración propia.

Figura 8.

Redes de colaboración por países

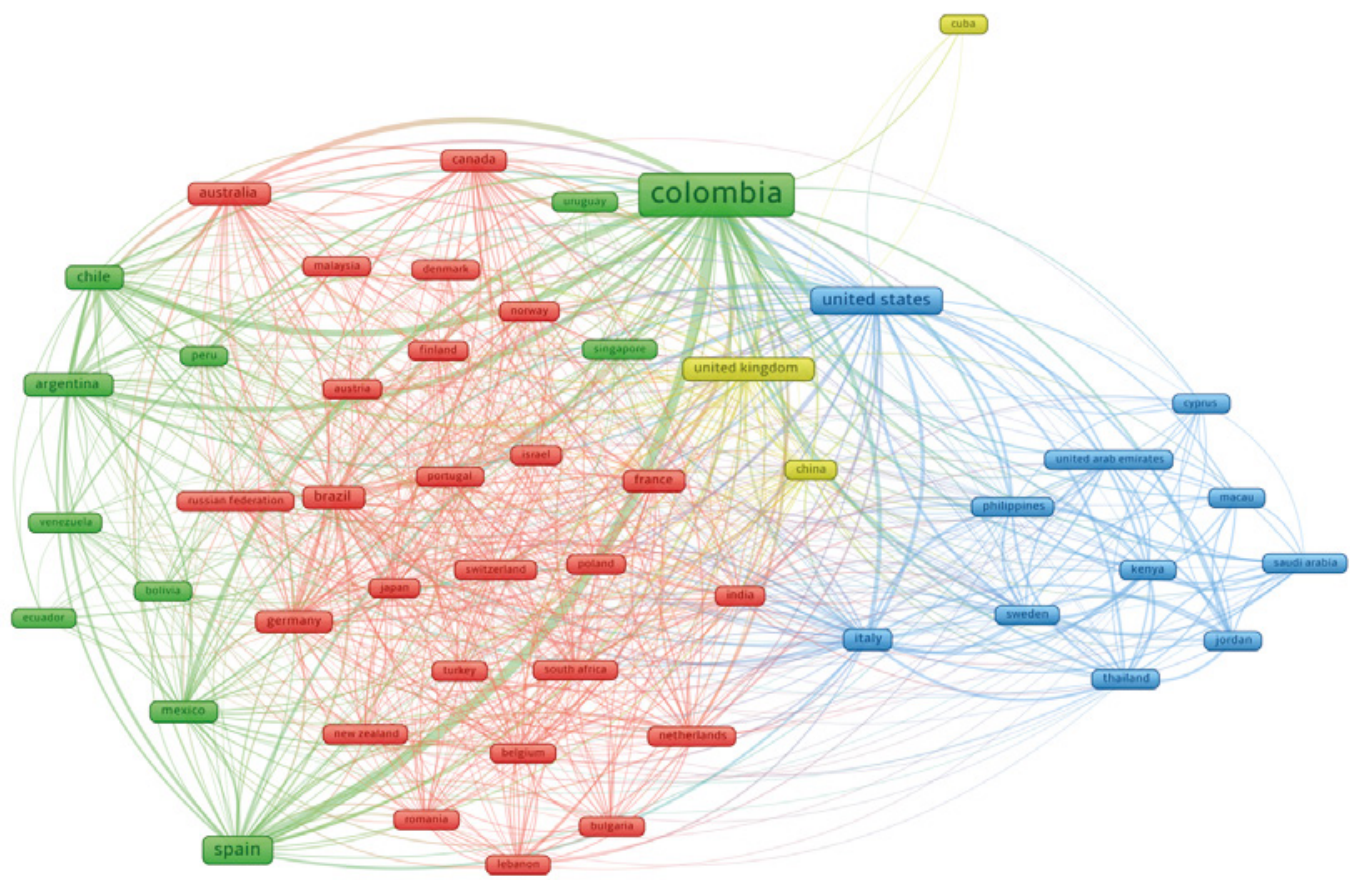

Nota. Fuente: elaboración propia. 
Tabla 8.

Top 10 de los países que más colaboran en la investigación psicológica en Colombia

\begin{tabular}{lccc}
\hline \multicolumn{1}{c}{ País } & Artículos & Citaciones & \% producción \\
\hline España & 256 & 2741 & 8.3 \\
Estados Unidos & 245 & 6142 & 8.0 \\
Chile & 98 & 1063 & 3.2 \\
Inglaterra & 79 & 1824 & 2.6 \\
Australia & 70 & 1154 & 2.3 \\
Argentina & 66 & 814 & 2.1 \\
Brasil & 64 & 1211 & 2.1 \\
México & 57 & 930 & 1.9 \\
Italia & 50 & 1301 & 1.6 \\
Francia & 47 & 889 & 1.5 \\
\hline
\end{tabular}

Nota. Fuente: elaboración propia.

Finalmente, es importante destacar que el trabajo colaborativo representa una práctica esencial en el desarrollo de las dinámicas de investigaciones al interior de diferentes comunidades académicas, y en el caso de la psicología colombiana no es distinto. Como vemos, las redes de colaboración representan en muchos casos un recurso importante para comprender las dinámicas de investigación que se establecen en una comunidad académica (Abdill et al., 2020; Bozeman \& Corley, 2004; Gutiérrez \& LandeiraFernández, 2018; Luukkonen et al., 1992).

\section{Discusión}

En general, los resultados del presente trabajo señalan que tanto la producción académica como los demás indicadores en los ecosistemas de conocimiento han aumentado en los últimos 15 años, lo cual ha ido de la mano con la creación de programas de posgrado en psicología en Colombia. Sin embargo, se encuentra que el aumento en la producción no se deriva directamente del conocimiento dentro de los programas de doctorado, por lo menos no en lo que se refleja en el número y tipo de publicaciones indexadas y lo reportado por los autores en su CvLAC. Esta gran diferencia en los reportes de bases de datos internacionales y el sistema local de producción (CvLAC) sugiere que existe una producción invisible internacionalmente. Acorde con esta diferencia, la gran cantidad de tesis de posgrado reportadas por los autores no es equivalente a la cantidad de artículos publicados, lo que sugiere que no existe la idea de investigación-publicación como eje fundamental de los programas de posgrado - maestría y doctorado-, incluidos los de doctorado en psicología. Esta situación indica que existe un vacío en la formación doctoral, ya que, al no estar centrada en los procesos de investigación y publicación, el objetivo final de una formación de alto nivel no se está cumpliendo.

Una explicación para estos hallazgos puede encontrarse en la forma en que aparece la formación doctoral en nuestro medio. Como afirman distintos autores, la formación doctoral en psicología en Colombia surgió en respuesta a la necesidad de fortalecer la disciplina al interior del país, tanto como profesión y como campo especializado de investigación (Alzate Medina, 2008; Cudina et al., 2017; Hayes, 1996; López-López et al., 2018); y ese hecho es el resultado del impulso de políticas de educación superior para estimular la acreditación de programas académicos para avanzar hacia el compromiso de una formación académica de calidad y de alto nivel (Bucheli et al., 2012; Chavarro et al., 2010; González-Zabala et al., 2017). Ahora bien, aunque este empuje administrativo tiene como resultado un crecimiento en la cantidad de programas, la desarticulación de los denominados ecosistemas de conocimiento refleja falencias durante en el proceso de formación que no pueden ser subsanadas únicamente con la creación del programa, sino que responden más a la cultura de investigación, a la baja promoción de la investigación, y a la escasa visibilización de tal investigación en los canales de comunicación de alta calidad - es decir, revistas internacionales o nacionales de reconocimiento internacional-.

Las tendencias actuales de las instituciones de educación superior en el ámbito internacional han sido testigo del rápido aumento de los títulos de doctorado profesional como un compromiso entre el impulso de niveles más altos de calificación en psicología, las presiones económicas en cuanto a los costos de formación y el deseo de que las universidades atraigan a los estudiantes (Brown et al., 2001). En el caso de países con formación doctoral de alto nivel, la relación entre investigación y aumento en la financiación es una relación directa que tiene como resultado una alianza que favorece la formación, la investigación y la financiación (Benavente et al., 2012; Graddy-Reed et al., 2021; Horta et al., 2016; Torka, 2018). En estos países, la financiación es determinante dentro del aumento de la producción de nuevo conocimiento tanto a nivel básico 
como aplicado, y abarca un amplio espectro en todas las áreas de conocimiento (Packalen \& Bhattacharya, 2020).

Aunque este modelo de inversión en formación de doctores corresponde a países de mayores ingresos, existen iniciativas importantes para el aumento en la financiación de doctores a nivel nacional que buscan ampliar e incentivar esta formación en investigación (Minciencias, 2017, 2021a, 2021b; Orozco et al., 2013). Actualmente, el impacto de este tipo de programas no ha sido evaluado, sin embargo, el aumento en la oferta de programas de posgrado, el aumento en la solicitud de admisión en los programas y las presiones del medio académico y profesional son indicadores indirectos de la estimulación de formación posgradual.

Por otra parte, un fenómeno asociado a una necesidad en formación complementaria que podría ir en detrimento de la equidad y los niveles adecuados de investigación y publicación es lo que se ha llamado créditos educativos, relacionado con el aumento en la carga de financiación por parte de los estudiantes. Respecto a este punto, en estudios desarrollados en Colombia se ha sugerido que estos programas de crédito, en vez de mejorar e incentivar la formación, lo que hacen es generar amplias brechas de desigualdad y disminución de oportunidades de estudiantes de menor clasificación socioeconómica (Gómez \& Celis, 2009). Sin embargo, no existen estudios recientes sobre el impacto en la financiación y crédito de los programas de posgrado en Colombia.

Retomando el aumento en los niveles de investigación, en el caso colombiano la psicología se ha expandido desde su fuerte tradición de investigación académica para asumir un papel cada vez más destacado como profesión (Ardila, 2019). No obstante, aunque la formación y la práctica de la psicología adquieren cada vez más importancia tanto para el psicólogo individual como para la disciplina en su conjunto (Kapplenbach, 2015), esto no se ve reflejado en los indicadores internacionales.

Por otra parte, destacan las alianzas nacionales, pero sobre todo regionales, como determinantes en el crecimiento y fortalecimiento de las comunidades académicas (Ávila-Toscano et al., 2019; Confalonieri et al., 2012). Reconociendo que nuestra región presenta condiciones, problemas y situaciones particulares, en ecosistemas comunes la cooperación ha sido una herramienta para crecer como comunidad. Parte de esta cooperación se ha fundamentado en la creación de organizaciones académicas de América
Latina e Iberoamérica que propenden por una alianza estratégica en investigación, producción y fortalecimiento de la disciplina, los cuales no solo dan respuesta a los retos de la disciplina, sino que también sirven de soporte para el crecimiento de los editores y el reconocimiento de los investigadores (López-López et al., 2021). Este tipo de alianzas e impulsos se acercan más a la idea de ecosistema de conocimiento, en donde diferentes actores soportan el proceso de investigación derivado de una articulación de los diferentes actores.

Adicional a esto, las dinámicas de investigación están mostrando un patrón de producción académica acelerado hacia la internacionalización de la investigación en psicología en Colombia. Esta práctica se acentúa en instituciones de educación superior provenientes del sector privado, probablemente como una respuesta a los procesos de globalización y a los procesos de acreditación que han propendido por el aumento en esta dinámica. Junto con este aumento, una característica de las revistas y difusión en América Latina es el modelo de acceso abierto, que responde a las necesidades de acceso a la información y a los bajos recursos que se requieren para publicar en los modelos particulares de acceso abierto (Alperin et al., 2015; Alperin \& Rozemblum, 2017). En este modelo, algunos estudios han mostrado que existe una ventaja en la citación respecto a revistas que tienen un modelo de pago por el acceso, siendo este el modelo tradición en Norteamérica y Europa, controlado por las grandes casas editoriales (Bautista-Puig et al., 2020; Sala et al., 2017). Esto llevaría a sugerir que una forma de trabajar para el fortalecimiento regional sería el aumento de los niveles de citación, con el cual se estaría visibilizando, reconociendo y valorando el trabajo de pares investigadores. Sin embargo, no conocemos estudios que den cuenta del nivel de citación entre investigadores de América Latina e Iberoamérica.

Finalmente, es importante destacar que cada vez son más y mejores las métricas en la medición de la producción, difusión e impacto de la productividad académica, con lo cual se ha trascendido más allá de la citación como el elemento central en la discusión de apropiación de conocimiento. Varios esfuerzos regionales han propuesto el uso de mediciones complementarias entre sí que den un mejor entendimiento de este proceso, con el fin de incentivar el trabajo de visibilización y uso de la investigación en psicología. 


\section{Referencias}

Abdill, R. J., Adamowicz, E. M., \& Blekhman, R. (2020). International authorship and collaboration across biorxiv preprints. ELife, 9, 1-17. https://doi.org/10.7554/ eLife.58496

Abramo, G., Cicero, T., \& D'Angelo, C. A. (2011). Assessing the varying level of impact measurement accuracy as a function of the citation window length. Journal of Informetrics, 5(4), 659-667. https://doi.org/10.1016/j. joi.2011.06.004

Acevedo-Triana, C., Torres, M., Aguilar-Bustamante, M. C., Hurtado-Parrado, C., Silva, L. M., \& López-López, W. (2018). Productivity analysis and impact of colombian psychology journals between 2000 and 2016. Revista Latinoamericana de Psicologia, 50(3), 145-159. https://doi.org/10.14349/rlp.2018.v50.n3.2

Aguado-López, E., Becerril-García, A., \& Aguilar Bustamante, M. (2016). Universitas Psychologica: un camino hacia la internacionalización. Universitas Psychologica, 15(2), 321-338. https://doi.org/10.11144/ Javeriana.upsy15-2.upci

Aguado-López, E., López-López, W., Becerril-García, A., \& Salas, G. (2017). Patrones de Internacionalización en Psicología desde la revista Interamericana de Psicología. Revista Interamericana de Psicología, 51(3), 268-281. https://journal.sipsych.org/index.php/IJP/article/view/ 897/pdf

Aguado-López, E., Rogel-Salazar, R., Garduño-Oropeza, G., Becerril-García, A., Zúñiga-Roca, M. F., \& VelázquezÁlvarez, A. (2009). Patrones de colaboración científica a partir de redes de coautoría. Convergencia Revista de Ciencias Sociales, 16, 225-258. https://www.redalyc. org/pdf/105/10512244010.pdf

Aguilar Bustamante, M. C. (2018). La necesaria discusión sobre las consecuencias de la Cienciometría sobre la construcción de las políticas de Ciencia y Tecnología. Diversitas, 14(1), 11-12. https://revistas.usantotomas.edu.co/index.php/diver sitas/article/view/4461

Aguilar Bustamante, M. C., \& Aguado López, E. (2018). Diversitas: Perspectivas en Psicología. Un camino de calidad y visibilidad de la disciplina para Colombia y el mundo. Diversitas, 14(1), 163-186. https://doi. org/10.15332/s1794-9998.2018.0001.12

Alperin, J. P. (2014). South America: Citation databases omit local journals. Nature, 511(7508), 155-155. https:// doi.org/10.1038/511155c

Alperin, J. P. (2015). Geographic variation in social media metrics: an analysis of Latin American journal articles. Aslib Journal of Information Management, 67(3), 289304. https://doi.org/10.1108/AJIM-12-2014-0176

Alperin, J. P., \& Rozemblum, C. (2017). La reinterpretación de visibilidad y calidad en las nuevas políticas de evaluación de revistas científicas. Revista Interamericana de Bibliotecología, 40(3), 231-241. https://doi.org/10.17533 /udea.rib.v40n3a04

Alperin, J. P., Babini, D., Chan, L., Gray, E., Guédon, J.C., Joseph, H., Rodrigues, E., Shearer, K., \& Vessuri, H. (2015). Open Access in Latin America: a Paragon for the Rest of the World. The Winnower. https://www.acade mia.edu/45326154/Open_Access_in_Latin_America_a Paragon for the Rest of the World

Alzate Medina, G. M. (2008). Efectos de la acreditación en el mejoramiento de la calidad de los programas de psicología de Colombia. Universitas Psychologica, 7(2), 425-439. https://revistas.javeriana.edu.co/index.php/revPsycho /article/view/425

Ana, J., Koehlmoos, T., Smith, R., \& Yan, L. L. (2013). Research Misconduct in Low- and Middle-Income Countries. PLoS Medicine, 10(3), 1-6. https://doi. org/10.1371/journal.pmed.1001315

Ardila, R. (2019). Historia de la Psicología en Colombia (2. ${ }^{\mathrm{a}}$ ed). Manual Moderno. 
Armayones Ruiz, M., Boixadós, M., Gómez Zúñiga, B., Guillamón, N., Hernández, E., Nieto, R., Pousada, M., \& Sara, B. (2015). Psicología 2.0: oportunidades y retos para el profesional de la psicología en el ámbito de la salud. Papeles del Psicólogo, 36(2), 153-160. http://www. papelesdelpsicologo.es/pdf/2571.pdf

Ávila-Toscano, J. H., Marenco-Escuderos, A. D., \& RomeroPérez, I. K. (2019). Redes de cooperación entre autores e instituciones en Ciencias Sociales dentro del modelo científico colombiano: comparación por género y área del conocimiento. Revista General de Información y Documentación, 29(1), 209-227. https://doi.org/10.5209/ rgid. 64545

Ávila-Toscano, J. H., \& Rambal-Rivaldo, L. I. (2020). Producción científica de investigadores de psicología del sistema científico colombiano según su clasificación y sexo. Avances en Psicología Latinoamericana, 38(3), 1-14. https://doi.org/10.12804/revistas.urosario.edu.co/ apl/a.8133

Ávila-Toscano, J. H., Marenco-Escuderos, A., \& Madariaga-Orozco, C. (2013). Indicadores bibliométricos, redes de coautorías y colaboración institucional en revistas colombianas de psicología. Avances en Psicología Latinoamericana, 32(1), 167-182. https://doi. org/10.12804/apl32.1.2014.12

Ávila-Toscano, J. H., Marenco-Escuderos, A., \& Orozco, C. M. (2014). Indicadores bibliométricos, Redes de Coautorías y colaboración institucional en revistas Colombianas de psicología. Avances en Psicología Latinoamericana, 32, 167-182. https://doi.org/10.12804/ ap132.1.2014.12

Ayodele, F. O., Yao, L., \& Haron, H. (2019). Promoting Ethics and Integrity in Management Academic Research: Retraction Initiative. Science and Engineering Ethics, 25(2), 357-382. https://doi.org/10.1007/s11948 -017-9941-z

Bajwa, N. ul H., \& König, C. J. (2019). How much is research in the top journals of industrial/organizational psychology dominated by authors from the
U.S.? Scientometrics, 120(3), 1147-1161. https://doi. org/10.1007/s11192-019-03180-2

Banco Mundial. (2021). Datos de libre acceso del Banco Mundial. https://datos.bancomundial.org

Barbosa, J., Barbosa, J., \& Rodríguez, M. (2013). Revisión y análisis documental para estado del arte: una propuesta metodológica desde el contexto de la sistematización de experiencias educativas. Investigación Bibliotecológica, 27(61), 83-105. http://www.scielo.org.mx/pdf/ib/ v27n61/v27n61a5.pdf

Bautista-Puig, N., Lopez-Illescas, C., de Moya-Anegon, F., Guerrero-Bote, V., Moed, H. (2020). Do journals flipping to gold open access show an OA citation or publication advantage? Scientometrics, 124(3), 2551-2575. https:// doi.org/10.1007/s11192-020-03546-X

Benavente, J. M., Crespi, G., Figal Garone, L., \& Maffioli, A. (2012). The impact of national research funds: A regression discontinuity approach to the Chilean FONDECYT. Research Policy, 41(8), 1461-1475. https://doi.org /10.1016/J.RESPOL.2012.04.007

Bornmann, L., Mutz, R., Haunschild, R., de Moya-Anegon, F., Clemente, M. de A. M., \& Stefaner, M. (2021). Mapping the impact of papers on various status groups: A new excellence mapping tool based on citation and reader scores. https://arxiv.org/abs/2103.10225v1

Bozeman, B., \& Corley, E. (2004). Scientists' collaboration strategies: implications for scientific and technical human capital. Research Policy, 33(4), 599-616. https:// doi.org/10.1016/j.respol.2004.01.008

Bucheli, V., Díaz, A., Calderón, J. P., Lemoine, P., Valdivia, J. A., Villaveces, J. L., \& Zarama, R. (2012). Growth of scientific production in Colombian universities: An intellectual capital-based approach. Scientometrics, 91(2), 369-382. https://doi.org/10.1007/s11192-012-0627-7

Bustos-González, A. (2019). Tránsito de universidad docente a universidad de investigación. ¿Un problema de información académica, de taxonomías o de rankings 
universitarios? El Profesional de La Información, 28(4), 1-13. https://doi.org/10.3145/epi.2019.jul.22

Brown, P., Green, A., \& Lauder, H. (2001). Globalization, Competitiveness, and Skill Formation. Oxford University Press.

Calderón-Prada, S., \& Cuartas-Arias, J. M. (2012). Visibilización de la producción académico-investigativa en psicología y "glocalización" de las capacidades productivas de la psicología en Colombia. Revista Colombiana de Psicologia, 21(1), 125-149. https://revis tas.unal.edu.co/index.php/psicologia/article/download $/ 31036 / 31106 / 112399$

Carlsson, B. (2006). Internationalization of innovation systems: A survey of the literature. Research Policy, 35(1), 56-67. https://doi.org/10.1016/j.respol.2005.08.003

Chavarro, D., Orozco, L. A., \& Villaveces, J. L. (2010). Análisis del perfil de los grupos de referencia del país. En J. L. Villaveces-Cardoso, R. Bonilla-Jiménez, V. A. Bucheli- Guerrero, D. A. Chavarro-Bohórquez, L. Delgado- Vanegas, C. Montilla-Vargas, L. A. OrozcoCastro, C. F. Ruiz-Ramos, \& R. Zarama- Urdaneta (Eds.), La investigación en Uniandes 2010. Construcción de una política (pp. 107-117). Ediciones Uniandes.

Confalonieri, L., Pagnoni, G., Barsalou, L. W., Rajendra, J., Eickhoff, S. B., \& Butler, A. J. (2012). Brain Activation in Primary Motor and Somatosensory Cortices during Motor Imagery Correlates with Motor Imagery Ability in Stroke Patients. International Scholarly Research Network. ISRN Neurology, 2012, 613595. https://doi. org/10.5402/2012/613595

Cronin, B. (2001). Hyperauthorship: A postmodern perversion or evidence of a structural shift in scholarly communication practices? Journal of the American Society for Information Science and Technology, 52(7), 558-569. https://doi.org/10.1002/asi.1097

Cudina, J. N., Millan, J. D., \& Ossa, J. C. (2017). Redes de comunicación científica en la investigación psicológica de las américas a través de la Revista Interamericana de Psicología. Revista Interamericana de Psicología, 51(3), 282-296. https://journal.sipsych.org/index.php/ IJP/article/view/898

Cudina, J. N., \& Ossa, J. C. (2016). The top 100 high-impact papers in Colombian psychology: A bibliometric study from WoS y Scopus. Informacao e Sociedade, 26(2), 137-154. https://periodicos.ufpb.br/ojs2/index.php/ies/ article/view/29200

Di Bitetti, M. S., \& Ferreras, J. A. (2017). Publish (in English) or perish: The effect on citation rate of using languages other than English in scientific publications. Ambio, 46(1), 121-127. https://doi.org/10.1007/ s13280-016-0820-7

Fernández, M. T., Gómez, I., Sebastían, J., \& Sebastián, S. (1998). La cooperación científica de los países de América Latina a través de indicadores bibliométricos. Interciencia, 23(6), 328-337. https://digital.csic.es/ handle/10261/247167

Forero, D. A., Trujillo, M. L., González-Giraldo, Y., \& Barreto, G. E. (2020). Scientific productivity in neurosciences in Latin America: a scientometrics perspective. International Journal of Neuroscience, 130(4), 398-406. https://doi.org/10.1080/00207454.2019.1692837

Franco-Suárez, O., \& Quevedo-Blasco, R. (2015). Análisis de las revistas iberoamericanas de Psicología y de Educación indexadas en el Journal Citation Reports del 2015. PSIENCIA. Revista Latinoamericana de Ciencia Psicológica, 9(4), 1-23. https://doi.org/10.5872/ psiencia/9.4.22

Frenken, K. (2002). A new indicator of European integration and an application to collaboration is scientific research. Economic Systems Research, 14(4), 345-361. https://doi. org/10.1080/0953531022000024833

Gallegos, M., Berra, M., Benito, E., \& López-López, W. (2014). Las nuevas dinámicas del conocimiento científico y su impacto en la Psicología Latinoamericana. Psicoperspectivas, 13(3), 106-117. 
https://doi.org/10.5027/psicoperspectivas-Vol13-Issue3 -fulltext-377

Gallegos, M., Pérez-Acosta, A. M., Klappenbach, H., López-López, W., \& Bregman, C. (2020). Los estudios bibliométricos en el campo de la psicología iberoamericana: Una revisión metabibliométrica. Interdisciplinaria Revista de Psicología y Ciencias Afines, 37(2), 95-115. https://doi.org/10.16888/ interd.2020.37.2.6

García-Martínez, A. T., Guerrero-Bote, V. P., \& MoyaAnegón, F. de. (2012). World Scientific Production in Psychology. Universitas Psychologica, 11(3), 699-717. https://revistas.javeriana.edu.co/index.php/revPsycho/ article/view/2439/2865

Garcia, A., Acevedo-Triana, C. A., \& López-López, W. (2015). The meaning of and proposals for LatinAmerican cooperation in psychology. Psykhe, 24(2). https://doi.org/10.7764/psykhe.24.2.765

García, A., Acevedo-Triana, C. A., \& López-López, W. (2014). Cooperación en las ciencias del comportamiento Latino Americanas: Una investigación documental. Terapia Psicológica, 32(2). https://doi.org/10.4067/ S0718-48082014000200009

García, A., López-López, W., Acevedo-Triana, C. A., \& Nogueira-Pereira, F. (2017). Cooperation in Latin America: the scientific psychology network. Diversitas, 13(1), 123-133. https://doi.org/10.15332/ s1794-9998.2017.0001.9

Garfield, E. (1992). Psychology Research, 1986-1990: A Citationist Perspective on the Highest Impact Papers, Institutions and Authors. Current Contents, 15(41), 155165. http://garfield.library.upenn.edu/essays/v15p155y 1992-93.pdf

Giraldo, B., \& Rodríguez, O. R. (2000). Eventos significativos de la psicología colombiana en el siglo XX. Suma Psicológica, 7(2), 275-294. http://publicaciones.konrad lorenz.edu.co/index.php/sumapsi/article/view/198
Glänzel, W. (2001). National characteristics in international scientific co-authorship relations. Scientometrics, 51(1), 69-115. https://doi.org/10.1023/A:1010512628145

Godin, B., \& Gingras, Y. (2000). The place of universities in the system of knowledge production. Research Policy, 29(2), 273-278. https://doi.org/10.1016/ S0048-7333(99)00065-7

Gómez, V. M., \& Celis, J. E. (2009). Crédito educativo, acciones afirmativas y equidad social en la educación superior en Colombia. Revista de Estudios Sociales, 106-117. https://www.redalyc.org/articulo.oa?id=81511781010

Gómez, Y. (2017). El baile de los que sobran: cambio cultural y evaluación académica. Revista Colombiana de Antropología, 53(2), 15-25. https://doi. org/10.22380/2539472X.115

González-Zabala, M. P., Galvis-Lista, E. A., \& AnguloCuentas, G. L. (2017). Análisis de indicadores de Ciencia, Tecnología e Innovación (CTI) propuestos por Instituciones de Educación Superior Latinoamericanas. Revista Virtual Universidad Católica del Norte, 38(60), 23-40. https://www.revistaespacios.com/a17v38n60/a17 v38n60p05.pdf

Graddy-Reed, A., Lanahan, L., \& D’Agostino, J. (2021). Training across the academy: The impact of $R \& D$ funding on graduate students. Research Policy, 50(5), 104224. https://doi.org/10.1016/J.RESPOL.2021.104224

Guerrero, J., \& Jaraba, B. (2009). La producción científica de la psicología colombiana: un análisis bibliométrico de las revistas académicas, 1949-2008. ASCOFAPSI.

Gutiérrez, G., \& Landeira-Fernández, J. (2018). Psychological research in Latin America: Current and future perspectives. En R. Ardila (Ed.), Psychology in Latin America (pp. 7-26). Springer. https://doi. org/10.1007/978-3-319-93569-0_2

Harzing, A. W. (2008). On becoming a high impact journal in international business and management. European 
Journal of International Management, 2(2), 115-118. https://doi.org/10.1504/EJIM.2008.017763

Hayes, N. (1996). What Makes a Psychology Graduate Distinctive? European Psychologist, 1(2), 130-134. https://doi.org/10.1027/1016-9040.1.2.130

Horta, H., Cattaneo, M., \& Meoli, M. (2016). PhD funding as a determinant of $\mathrm{PhD}$ and career research performance. Studies in Higher Education, 43(3), 542-570. https:// doi.org/10.1080/03075079.2016.1185406

Jaraba-Barrios, B., Guerrero-Castro, J., Jack GómezMorales, Y., \& López-López, W. (2011). Bibliometría e historia de las prácticas académicas locales: un esbozo a partir del caso de la psicología en Colombia. Avances en Psicología Latinoamericana, 29(2), 354-369. https:// revistas.urosario.edu.co/index.php/apl/article/view/2008

Jonkers, K., \& Tijssen, R. (2008). Chinese researchers returning home: Impacts of international mobility on research collaboration and scientific productivity. Scientometrics, 77(2), 309-333. https://doi.org/10.1007/ s11192-007-1971-x

Kamler, B. (2008). Rethinking doctoral publication practices: Writing from and beyond the thesis. Studies in Higher Education, 33(3), 283-294. https://doi. org/10.1080/03075070802049236

Klappenbach, H. (2015). La formación universitaria en psicología en Argentina: perspectivas actuales y desafíos a la luz de la historia. Universitas Psychologica, 14(3), 937-960. http://dx.doi.org/10.11144/Javeriana.upsy14-3. fupa

Krabel, S., Siegel, D. S., \& Slavtchev, V. (2012). The internationalization of science and its influence on academic entrepreneurship. Journal of Technology Transfer, 37(2), 192-212. https://doi.org/10.1007/s10961-010-9182-7

Krampen, G. (2008). The evaluation of university departments and their scientists: Some general considerations with reference to exemplary bibliometric publication and citation analyses for a Department of psychology.
Scientometrics, 76(1), 3-21. https://doi.org/10.1007/ s11192-007-1900-z

Lafont, P. (2014). Knowledge Producing of the Doctoral Thesis: Between Scientific Utility and Social Usage. Procedia - Social and Behavioral Sciences, 116, 570577. https://doi.org/10.1016/j.sbspro.2014.01.259

Liberatore, G., \& Hermosilla, A. (2008). La Producción Científica Argentina en Psicología: Un Análisis de la Visibilidad e Impacto en el Ámbito Internacional y su Comparación con Países de la Región. Revista Interamericana de Psicología, 42(3), 507-512. https:// www.redalyc.org/articulo.oa? $\mathrm{id}=28442310$

López-López, W. (2018). Un cambio en la concepción de la edición académica. Del conocimiento centrado en los productos al conocimiento orientado a los servicios. Universitas Psychologica, 17(1), 1-2. http://www.scielo. org.co/pdf/rups/v17n1/1657-9267-rups-17-01-00001. pdf

López-López, W. (2019a). Ecosistema del conocimiento en América Latina: El acceso abierto, métricas, paradojas y contradicciones. Universitas Psychologica, 18(4), 1-3. https://doi.org/10.11144/Javeriana.upsy18-4.ecal

López-López, W. (2019b). La comunicación científica y la política pública: ¿Es posible una política pública basada en la evidencia? Universitas Psychologica, 18(1), 1-3. https://doi.org/10.11144/javeriana.upsy18-1.ccpp

López-López, W. (2020). La Coalición S y el Plan S: Implicaciones para los ecosistemas de conocimiento en América Latina. Universitas Psychologica, 19, 1-4. https://doi.org/10.11144/javeriana.upsy19.csps

López-López, W., Caycedo, C., Acevedo-Triana, C. A., Hurtado-Parrado, C., Silva, L. M., \& Aguilar-Bustamante, M. C. (2018). Training, Academic, and Professional development in Psychology in Colombia: Challenges and Perspectives. En G. J. Rich, A. Padilla-López, L. K. de Souza, L. Zinkiewicz, J. Taylor, \& J. L. S. Binti Jaafar (Eds.), Teaching Psychology around the World (Vol. 4, pp. 53-79). Cambridge Scholars Publishing. 
López-López, W., de Moya Anegón, F., Acevedo-Triana, C. A., Garcia, A., \& Silva, L. M. (2015). Psychological Research Collaboration and Visibility in Iberoamerica. Psicologia: Reflexão e Crítica, 28(Suppl 1), 72-81. https://doi.org/10.1590/1678-7153.20152840011

López-López, W., Silva, L. M., García-Cepero, M. C., Aguilar-Bustamante, M. C., \& Aguado, E. (2010). Panorama general de la producción académica en la psicología colombiana indexada en psicoredalyc. Acta Colombiana de Psicología, 13(2), 35-46. https://actaco lombianapsicologia.ucatolica.edu.co/article/view/368

López-López, W., Tortosa, F., Santolaya, F., Tortosa, M., Santolaya Prego de Oliver, J., \& Aguilar-Bustamante, M. C. (2021). History, difficulties, and challenges of the Ibero-American Federation of Psychology Associations. Papeles del Psicólogo, 42(1), 67-78. https://doi. org/10.23923/pap.psicol2021.2951

Luukkonen, T., Persson, O., \& Sivertsen, G. (1992). Understanding Patterns of International Scientific Collaboration. Science, Technology \& Human Values, 17(1), 101-126. https://doi. org/10.1177/016224399201700106

Miguel, S., Chinchilla-Rodríguez, Z., González, C., \& de Moya Anegón, F. (2012). Analysis and visualization of the dynamics of research groups in terms of projects and co-authored publications. A case study of library and information science in Argentina. Information Research, 17(3), 524. https://digital.csic.es/handle/10261/63799

Minciencias. (2017). Programa Colombia Cientifica aportará al desarrollo de la capacidad investigativa del país. Programa Colombia Científica. https://bit.ly/2Y71FhN

Minciencias. (2021a). Convocatoria del Fondo de Ciencia, Tecnología e Innovación del Sistema General de Regalías para la conformación de una lista de proyectos elegibles para ser viabilizados, priorizados y aprobados por el OCAD en el marco del Programa de Becas de Excelencia. Plan Bienal de Convocatorias. https://bit. ly/3k1jAM9
Minciencias. (2021b). Objetivo del Programa de Formación de Alto Nivel. Minciencias. https://bit.ly/3jWWPZC

Ministerio de Educación. (2019). Sistema de aseguramiento de la calidad de la educación superior. https://bit. ly/3jZN7WC

Morales, Y. J. G., Jaraba-Barrios, B., Guerrero-Castro, J., \& López-López, W. (2012). Entre internacionalización y consolidación de comunidades académicas locales. Sobre la revista latinoamericana de psicología. Revista Colombiana de Psicologia, 21(1), 97-110. https://reposi torio.unal.edu.co/handle/unal/41688

Moya Anegon, F. De. (2011). Informe de diagnóstico sobre la producción científica de Colombia (Scopus, 20032010). En Producción científica de Colombia. Scopus, 2003-2010. Minciencias. http://repositorio.minciencias. gov.co/handle/11146/485

Orozco, L. A., Ruiz, C. F., Bonilla, R., \& Chavarro, D. (2013). Los grupos de investigación en Colombia. Sus prácticas, su reconocimeinto y su legitimidad. En M. Salazar (Ed.), Colciencias cuarenta años: entre la legitimidad, la normatividad y la práctica (pp. 634-687). Observatorio Colombiano de Ciencia y Tecnología.

Ossa, J. C., \& Cudina, J. N. (2016). Sesenta años de las revistas de psicología en Colombia. Revista Guillermo de Ockham, 14(2), 7-17. https://doi.org/10 $.21500 / 22563202.2720$

Otte, E., \& Rousseau, R. (2002). Social network analysis: A powerful strategy, also for the information sciences. Journal of Information Science, 28(6), 441-453. https:// doi.org/10.1177/016555150202800601

Packalen, M., \& Bhattacharya, J. (2020). NIH funding and the pursuit of edge science. Proceedings of the National Academy of Sciences, 117(22), 12011-12016. https://doi. org/10.1073/pnas.1910160117

Peña-Correal, T. (1993). La psicología en Colombia: Historia de una disciplina y una profesión. En C. E. 
Vasco, D. Obregón, \& L. E. Orozo (Eds.), Historia social de la ciencia en Colombia, Tomo IX: ciencias sociales (pp. 95-179). Colciencias.

Piwowar, H., Priem, J., Larivière, V., Alperin, J. P., Matthias, L., Norlander, B., Farley, A., West, J., \& Haustein, S. (2018). The state of OA: a large-scale analysis of the prevalence and impact of Open Access articles. PeerJ, 6, e4375. https://doi.org/10.7717/peerj.4375

Polanco-Carrasco, R., Gallegos, M., Salas, G., \& LópezLópez, W. (2017). Las revistas de psicología en Chile: historia y situación actual. Terapia Psicológica, 35(1), 8193. https://doi.org/10.4067/S0718-48082017000100008

Polanco, F., Beria, J., \& Klappenbach, H. (2017). Cinco décadas de la Revista Interamericana de Psicología. Un estudio socio-bibliométrico. Revista Interamericana de Psicología, 51(3), 297-319. http://doi.org/10.30849/rip/ijp.v51i3.910

Puche-Navarro, R., \& Ossa, J. C. (2012). Claves de la publicación psicológica en Colombia: Ritmo, grupos y modalidades de producción en la práctica investigativa. Revista Colombiana de Psicología, 21(1), 79-95. http:// www.scielo.org.co/pdf/rcps/v21n1/v21n1a06.pdf

Ravelo-Contreras, E. L., Barboza-Palomino, M., Mejía, S., Millán, J. D., \& Salas, G. (2020). La producción científica de la revista Acta Colombiana de Psicología: Análisis descriptivo y bibliométrico del período 2015-2019. Acta Colombiana de Psicología, 23(2), 170-204. https://doi. org/10.14718/acp.2020.23.2.8

Rivera-Garzón, D. M. (2008). Caracterización de la comunidad científica de Psicología que publica en la revista Universitas Psychologica (2002-2008). Universitas Psychologica, 7(3), 917-932. http://revistas.javeriana. edu.co/index.php/revPsycho/article/download/401/283.

Robayo-Castro, B., Rico, J. L., Hurtado-Parrado, C., \& Ortega, L. A. (2016). Impacto y calidad de la productividad académica de los investigadores en Colombia en neurociencia comportamental utilizando modelos animales. Universitas Psychologica, 15(5). https://doi.org/10.11144/Javeriana.upsy15-5.icpa
Romero-Torres, M., Acosta-Moreno, L. A., \& TejadaGómez, M.-A. (2013). Ranking de revistas científicas en Latinoamérica mediante el índice h: estudio de caso Colombia. Revista Española de Documentación Cientifica, 36(1), e003. https://doi.org/10.3989/redc .2013.1.876

Russell, J. M., Ainsworth, S., Del Río, J. A., NarváezBerthelemot, N., \& Cortés, H. D. (2007). Colaboración científica entre países de la región latinoamericana. Revista Española de Documentación Científica, 30(2), 180-198. https://doi.org/10.3989/redc.2007.v30.i2.378

Sala, F. G., Lluch, J. O., Gil, F. T., \& Ortega, M. P. (2017). Characteristics of monographic special issues in IberoAmerican psychology journals: visibility and relevance for authors and publishers. Scientometrics, 112(2), 10691077. https://doi.org/10.1007/s11192-017-2372-4

Salas, G., Vega-Arce, M., González, C., Ossa, J. C., Cudina, J. N., Caycho-Rodríguez, T., Barboza-Palomino, M., Ventura-León, J., Guerra-Labbé, L., \& López-López, W. (2019). The fiftieth anniversary of the Revista latinoamericana de psicología: A bibliometric analysis. Revista Latinoamericana de Psicología, 51(2), 123-135. https:// doi.org/10.14349/rlp.2019.v51.n2.7

Salazar-Acosta, M., Lucio-Arias, D., López-López, W., \& Aguado-López, E. (2013). Informe sobre la producción cientifica de Colombia en revistas iberoamericanas de acceso abierto en redalyc.org, 2005-2011 (1. ${ }^{\text {a }}$ ed.). Universidad Autónoma del Estado de México.

Torka, M. (2018). Projectification of Doctoral Training? How Research Fields Respond to a New Funding Regime. Minerva, 56(1), 59-83. https://doi.org/10.1007/ S11024-018-9342-8

Van Eck, N. J., \& Waltman, L. (2010). Software survey: VOSviewer, a computer program for bibliometric mapping. Scientometrics, 84(2), 523-538. https://doi. org/10.1007/s11192-009-0146-3

Vasconcelos, S., Leta, J., Costa, L., Pinto, A., \& Sorenson, M. M. (2009). Discussing plagiarism in Latin American 
science: Brazilian researchers begin to address an ethical issue. EMBO Reports, 10(7), 677-682. https://doi. org/10.1038/embor.2009.134

Vasconcelos, S., Sorenson, M. M., Leta, J., Sant'Ana, M. C., \& Batista, P. D. (2008). Researchers'writing competence: a bottleneck in the publication of Latin-American science? EMBO Reports, 9(8), 700-702. https://doi. org/10.1038/embor.2008.143

Vélez-Cuartas, G., Gómez-Flórez, H., Úsuga-Ciro, A., \& Vélez-Trujillo, M. (2014). Diversidad y reconocimiento de la producción académica en los sistemas de evaluación de la investigación en Colombia. Revista Española de Documentación Científica, 37(3), 1-14. https://doi.org/10.3989/redc.2014.3.1133
Vera-Villarroel, P., López-López, W., Lillo, S., Silva, L. M. (2011). La producción científica en psicología latinoamericana: Un análisis de la investigación por países. Revista Latinoamericana de Psicología, 43(1), 95-104. http://www.scielo.org.co/pdf/rlps/v43n1/v43n1a08.pdf

Vessuri, H. (2014). Cambios en las ciencias ante el impacto de la globalización. Revista de Estudios Sociales, 50(50), 167-173. https://doi.org/10.7440/res50.2014.16

White, M. J., \& White, K. G. (1977). Citation analysis of psychology journals. American Psychologist, 32(5), 301305. https://doi.org/10.1037/0003-066X.32.5.301 
Tabla suplementaria 1.

Top 15 autores más productivos de la psicología en Colombia según datos de Scopus

\begin{tabular}{|c|c|c|c|c|c|c|c|c|}
\hline Name & $\begin{array}{c}\text { Scopus } \\
\text { document }\end{array}$ & $\begin{array}{c}\text { Scopus } \\
\text { Citations }\end{array}$ & $\begin{array}{l}\text { Scopus } \\
\text { h-index }\end{array}$ & $\begin{array}{l}\text { Scholar } \\
\text { Citation }\end{array}$ & $\begin{array}{l}\text { Scholar } \\
\text { h_Index }\end{array}$ & RGcitation & Field & Institución \\
\hline López-López, Wilson & 56 & 377 & 13 & 4023 & 30 & 2512 & $\begin{array}{l}\text { Psicología } \\
\text { política }\end{array}$ & $\begin{array}{c}\text { Pontificia Universidad } \\
\text { Javeriana }\end{array}$ \\
\hline $\begin{array}{l}\text { Pérez-Acosta, } \\
\text { Andrés Manuel }\end{array}$ & 27 & 67 & 6 & 1246 & 18 & 524 & $\begin{array}{l}\text { Psicología } \\
\text { aplicada }\end{array}$ & Universidad del Rosario \\
\hline Ruiz, Francisco José & 24 & 199 & 14 & 2714 & 24 & 1551 & Psicología clínica & $\begin{array}{c}\text { Fundación Universitaria } \\
\text { Konrad Lorenz }\end{array}$ \\
\hline Vinaccia, Stefano & 22 & 130 & 10 & 5941 & 40 & 2124 & Psicología clínica & Universidad del Sinú \\
\hline $\begin{array}{l}\text { Quiceno, Japcy Mar- } \\
\text { garita }\end{array}$ & 18 & 125 & 8 & 3350 & 32 & 996 & Psicología clínica & Universidad de Medellín \\
\hline Barreto, Idaly & 17 & 78 & 6 & 924 & 17 & 415 & $\begin{array}{l}\text { Psicología } \\
\text { política }\end{array}$ & $\begin{array}{c}\text { Universidad Católica } \\
\text { de Colombia }\end{array}$ \\
\hline Ardila, Rubén & 16 & 35 & 7 & 7987 & 39 & 1082 & $\begin{array}{l}\text { Historia de la } \\
\text { psicología }\end{array}$ & $\begin{array}{c}\text { Universidad Nacional } \\
\text { de Colombia }\end{array}$ \\
\hline $\begin{array}{c}\text { Palacio Sañudo, Jorge } \\
\text { Enrique }\end{array}$ & 15 & 82 & 8 & 2871 & 30 & 869 & Psicología social & Universidad del Norte \\
\hline Vallejo-Medina, Pablo & 14 & 133 & 14 & 1066 & 19 & 641 & Psicología clínica & $\begin{array}{c}\text { Fundación Universitaria } \\
\text { Konrad Lorenz }\end{array}$ \\
\hline Gantiva, Carlos & 14 & 57 & 4 & 1501 & 18 & 276 & Psicología clínica & Universidad de los Andes \\
\hline Juárez, Fernando & 13 & 49 & 6 & 1640 & 22 & 525 & $\begin{array}{c}\text { Psicología } \\
\text { organizacional }\end{array}$ & Universidad del Rosario \\
\hline $\begin{array}{l}\text { Cuartas, Jorge } \\
\text { Mauricio }\end{array}$ & 13 & 12 & 6 & 438 & 7 & 394 & Neuropsicología & $\begin{array}{c}\text { Universidad de San } \\
\text { Buenaventura }\end{array}$ \\
\hline Cárdenas, F. P. & 12 & 23 & 8 & 805 & 12 & 496 & Psicología clínica & Universidad de los Andes \\
\hline Gutiérrez, Germán & 11 & 37 & 5 & 984 & 14 & 454 & $\begin{array}{l}\text { Psicología } \\
\text { aplicada }\end{array}$ & $\begin{array}{c}\text { Universidad Nacional } \\
\text { de Colombia }\end{array}$ \\
\hline $\begin{array}{c}\text { Garzón-Umerenkova, } \\
\text { Angélica }\end{array}$ & 11 & 67 & 6 & 279 & 10 & 118 & $\begin{array}{l}\text { Psicología } \\
\text { educativa }\end{array}$ & $\begin{array}{c}\text { Fundación Universitaria } \\
\text { Konrad Lorenz }\end{array}$ \\
\hline
\end{tabular}

Tabla suplementaria 2.

Top 5 autores más productivos por cada IES que oferta programas de doctorado en psicología, datos de Scopus y CvLAC

\begin{tabular}{ccccccc}
\hline Universidad & Investigador & Doc. & Citations & h-index & $\begin{array}{c}\text { Citations per } \\
\text { Publication }\end{array}$ & $\begin{array}{c}\text { Artículos } \\
\text { en CvLAC }\end{array}$ \\
\hline \multirow{3}{*}{$\begin{array}{c}\text { Pontificia Universidad } \\
\text { Javeriana }\end{array}$} & Wernando López-López & 59 & 326 & 13.0 & 5.5 & 142 \\
& Luis Manuel Silva & 8 & 86 & 5.0 & 7.5 & 32 \\
& Cesar Acevedo-Triana & 8 & 44 & 6.0 & 5.5 & 17 \\
& Hernán Camilo Pulido-Martínez & 7 & 16 & 4.0 & 2.3 & 26 \\
Universidad & Fernando Cardenas & 11 & 18 & 8.0 & 1.6 & 46 \\
de los Andes & William Jiménez-Leal & 10 & 17 & 3.0 & 1.7 & 13 \\
& Eric Quintane & 9 & 126 & 9.0 & 14.0 & 6 \\
& Ana Lucía Jaramillo-Sierra & 8 & 32 & 4.0 & 4.0 & 13 \\
& Enrique Chaux & 8 & 72 & 8.0 & 9.0 & 27 \\
Universidad Nacional & Rubén Ardila & 16 & 35 & 7.0 & 2.2 & 134 \\
de Colombia & Javier Alejandro Corredor Aristizábal & 9 & 2 & 1.0 & 0.2 & 29 \\
& Alejandro Rosas & 8 & 29 & 4.0 & 3.6 & 55 \\
& Germán Gutiérrez & 8 & 13 & 5.0 & 1.6 & 49 \\
& Marisol Rodríguez Lamprea & 7 & 25 & 10.0 & 3.6 & 33 \\
\hline
\end{tabular}




\begin{tabular}{|c|c|c|c|c|c|c|}
\hline Universidad & Investigador & Doc. & Citations & h-index & $\begin{array}{l}\text { Citations per } \\
\text { Publication }\end{array}$ & $\begin{array}{l}\text { Artículos } \\
\text { en CvLAC }\end{array}$ \\
\hline \multirow{5}{*}{$\begin{array}{c}\text { Fundación } \\
\text { Universitaria } \\
\text { Konrad Lorenz }\end{array}$} & Francisco José Ruiz & 35 & 225 & 14.0 & 6.4 & 74 \\
\hline & Pablo Vallejo-Medina & 14 & 120 & 14.0 & 8.6 & 51 \\
\hline & Idaly Barreto & 9 & 29 & 6.0 & 3.2 & 33 \\
\hline & Diana Marcela Delgado & 9 & 32 & 4.0 & 3.6 & 16 \\
\hline & Diana Riaño-Hernández & 8 & 66 & 5.0 & 8.3 & 11 \\
\hline \multirow{5}{*}{$\begin{array}{l}\text { Universidad } \\
\text { de Antioquia }\end{array}$} & David Antonio Pineda Salazar & 17 & 281 & 27.0 & 16.5 & 139 \\
\hline & Natalia Trujillo & 10 & 230 & 11.0 & 23.0 & 41 \\
\hline & Daniel Camilo Aguirre-Acevedo & 10 & 39 & 10.0 & 3.9 & 101 \\
\hline & Yamile Bocanegra & 7 & 160 & 8.0 & 22.9 & 14 \\
\hline & Mauricio Barrera-Valencia & 6 & 47 & 3.0 & 7.8 & 31 \\
\hline \multirow{5}{*}{$\begin{array}{c}\text { Universidad de } \\
\text { San Buenaventura }\end{array}$} & Carlos Gantiva & 15 & 60 & 4.0 & 4.0 & 55 \\
\hline & Japcy Margarita Quiceno & 14 & 117 & 8.0 & 8.4 & $*$ \\
\hline & Jorge Mauricio Cuartas & 12 & 9 & 6.0 & 0.8 & 37 \\
\hline & Liliana Maria Uribe Tirado & 11 & 261 & 10.0 & 23.7 & 52 \\
\hline & Stefano Vinaccia & 11 & 76 & 10.0 & 6.9 & 165 \\
\hline \multirow{5}{*}{ Universidad del Norte } & Jorge Enrique Palacio Sañudo & 14 & 83 & 9.0 & 5.9 & 93 \\
\hline & Oscar Oviedo-Trespalacios & 10 & 71 & 14.0 & 7.1 & 23 \\
\hline & Jorge Iván Vélez & 9 & 29 & 19.0 & 3.2 & 41 \\
\hline & Carlos José De Los Reyes Aragón & 5 & 25 & 4.0 & 5.0 & 26 \\
\hline & Camilo A. Madariaga & 5 & 14 & 2.0 & 2.8 & 56 \\
\hline \multirow{5}{*}{ Universidad del Valle } & Rebeca Puche-Navarro & 4 & 8 & 3.0 & 2.0 & 27 \\
\hline & Javier Murillo Muñoz & 3 & 12 & 2.0 & 4.0 & 1 \\
\hline & Andrés Fandiño-Losada & 3 & 8 & 6.0 & 2.7 & 48 \\
\hline & Óscar Sierra-Fitzgerald & 2 & 5 & 2.0 & 2.5 & 10 \\
\hline & Catalina Trujillo & 2 & 1 & 1.0 & 0.5 & 4 \\
\hline \multirow{5}{*}{$\begin{array}{c}\text { Universidad Católica } \\
\text { de Colombia }\end{array}$} & Constanza Londoño Pérez & 10 & 35 & 6.0 & 3.5 & 52 \\
\hline & Bertha Lucía Avendaño Prieto & 7 & 1 & 1.0 & 0.1 & 35 \\
\hline & Ernesto Ravelo-Contreras & 4 & 8 & 3.0 & 2.0 & $*$ \\
\hline & Ronald Toro & 3 & 2 & 2.0 & 0.7 & 32 \\
\hline & Delio Ignacio Castaneda & 3 & 24 & 6.0 & 8.0 & 33 \\
\hline \multirow{5}{*}{$\begin{array}{l}\text { Universidad de } \\
\text { la Costa }\end{array}$} & Tatiana Javier Cobo-Ibáñez & 10 & 60 & 26.0 & 6.0 & $*$ \\
\hline & Omar Fernando Cortés Peña & 5 & 6 & 2.0 & 1.2 & 34 \\
\hline & María del Mar Sánchez-Fuentes & 5 & 20 & 8.0 & 4.0 & 26 \\
\hline & Jesús Fernández-Lucas & 2 & 9 & 13.0 & 4.5 & $*$ \\
\hline & Carlos Alberto Jiménez Cabarcas & 2 & 0 & 0.0 & 0.0 & 3 \\
\hline \multirow{5}{*}{$\begin{array}{l}\text { Universidad } \\
\text { Santo Tomás }\end{array}$} & María Constanza Aguilar Bustamante & 4 & 21 & 3.0 & 5.3 & 27 \\
\hline & Luis Alberto Quiroga Baquero & 3 & 4 & 1.0 & 1.3 & 13 \\
\hline & Jorge Mario Jaramillo & 2 & 17 & 1.0 & 8.5 & 14 \\
\hline & María Isabel Rendón & 2 & 23 & 2.0 & 11.5 & 14 \\
\hline & Claudia Pineda-Marín & 2 & 2 & 5.0 & 1.0 & 24 \\
\hline \multirow{5}{*}{$\begin{array}{l}\text { Universidad } \\
\text { Simón Bolívar }\end{array}$} & Yolima Alarcón-Vásquez & 5 & 14 & 3.0 & 2.8 & 23 \\
\hline & Marly Johana Bahamón & 5 & 16 & 3.0 & 3.2 & 43 \\
\hline & Martha Martínez-Banfi & 3 & 2 & 1.0 & 0.7 & 10 \\
\hline & Wilmar Pineda-Alhucema & 3 & 17 & 2.0 & 5.7 & 10 \\
\hline & Johan E. Acosta-López & 3 & 16 & 3.0 & 5.3 & 15 \\
\hline
\end{tabular}

Nota. * CvLAC no disponible para visualización. 